\title{
Asymptotic normalization coefficients for mirror virtual nucleon decays in a microscopic cluster model
}

\author{
N. K. Timofeyuk ${ }^{1)}$ and P. Descouvemont ${ }^{2)}$ \\ 1) Department of Physics, University of Surrey, Guildford, Surrey GU2 7XH, England, UK \\ 2) Physique Nucléaire Théorique et Physique Mathématique, CP229 \\ Université Libre de Bruxelles, B1050 Brussels, Belgium
}

(Dated: September 17, 2018)

\begin{abstract}
It has been suggested recently (Phys.Rev.Lett. 91, 232501 (2003)) that charge symmetry of nucleon-nucleon interactions relates the Asymptotic Normalization Coefficients (ANCs) of proton and neutron virtual decays of mirror nuclei. This relation is given by a simple analytical formula which involves proton and neutron separation energies, charges of residual nuclei and the range of their strong interaction with the last nucleon. Relation between mirror ANCs, if understood properly, can be used to predict astrophysically relevant direct proton capture cross sections using neutron ANCs measured with stable beams. In this work, we calculate one-nucleon ANCs for several light mirror pairs, using microscopic two-, three- and four-cluster models, and compare the ratio of mirror ANCs to the predictions of the simple analytic formula. We also investigate mirror symmetry between other characteristics of mirror one-nucleon overlap integrals, namely, spectroscopic factors and single-particle ANCs.
\end{abstract}

PACS numbers: 21.60.Gx, 21.10.Jx, 27.20.+n, 27.30.+t

\section{INTRODUCTION}

The asymptotic normalization coefficient (ANC) for one-nucleon virtual decay $A \rightarrow(A-1)+N$ is one of the fundamental characteristics of a nucleus $A$. It determines the magnitude of the large distance behaviour of the projection of the bound state wave function of the nucleus $A$ onto the binary channel $(A-1)+N$. The recent interest in studying the one-nucleon ANCs is due to the role which they play in nuclear astrophysics for predicting cross sections of non-resonant capture reactions at stellar energies. The ANCs provide overall normalization of the astrophysical $S$-factors of such reactions. Since the same ANCs play a crucial role in other peripheral processes such as transfer reactions, they can be measured in laboratories and used to predict non-resonant capture processes at low stellar energies [1].

To determine relevant to astrophysics proton ANCs from transfer reactions, the use of radioactive beams is often required, which generally involves more difficult and less accurate experiments than those possible with stable beams. At the same time, stable beams can often be used to determine neutron ANCs associated with mirror virtual one-neutron decays. This has been noticed some time ago in Refs. 2, 3], where the one-nucleon ANCs of the mirror pairs ${ }^{8} \mathrm{~B}-{ }^{8} \mathrm{Li}$ and ${ }^{12} \mathrm{~N}-{ }^{12} \mathrm{~B}$ were studied in a microscopic approach. In these works, the calculated ANCs themselves depended strongly on the choice of the nucleon-nucleon (NN) force but the ratios of ANCs for mirror pairs were practically independent of the choice of the NN force. This property of the ANC ratios could be used to predict proton capture rates at astrophysical energies from information about mirror ANC's obtained from transfer reactions with stable beams. A first experiment which uses the idea of Ref. [2] to deduce the ANC of ${ }^{8} \mathrm{~B}$ from the ${ }^{8} \mathrm{Li}$ ANC has been already performed [4].
Recently, it has been pointed out that the ANCs for mirror virtual decays ${ }^{A} Z_{N} \rightarrow{ }^{A-1} Z_{N-1}+n$ and ${ }^{A} N_{Z} \rightarrow{ }^{A-1} N_{Z-1}+p$ are related if the charge symmetry of nucleon-nucleon (NN) interactions is satisfied [5]. This link is approximated by a simple analytical formula which is a consequence of the relation between the onshell amplitudes of mirror virtual decays. These on-shell amplitudes, called vertex constants, are equivalent to the coupling constants in particle physics [6].

A link between mirror ANCs also follows from the single-particle model of nuclei if charge symmetry is valid both for single-particle potential wells and for mirror onenucleon spectroscopic factors. As shown in Ref. [5], predictions of such a single-particle model are close to the predictions of the simple analytical formula, derived from consideration of mirror vertex constants, if nucleon separation energies are relatively large. This agreement deteriorates with decrease of separation energies and for weakly-bound $s$-states with nodes, the difference between the two different estimates for the ratio of mirror ANCs can reach $\sim 15-20 \%$.

At present, more accurate but simple approximations relating mirror ANCs are not available. Therefore, numerical calculations using theoretical structure models are very important. In the present paper, we try to improve our understanding of relation between mirror ANCs by performing calculations within a microscopic cluster model (MCM). This model considers the manybody nature of atomic nuclei and takes into account differences in nuclear structure arising because of charge symmetry breaking due to the Coulomb interaction. We expect that, in MCM, the lack of accuracy of the two different approximations from Ref. [5] is reduced. We calculate one-nucleon overlap integrals for some mirror light nuclei and concentrate mainly on mirror ANCs, but other properties of overlap integrals, such as spectroscopic fac- 
tors, r.m.s. radii and single-particle ANCs, and their mirror symmetry are investigated.

In Sec. II we give definitions for ANCs, their expressions via nuclear wave functions, show the approximations for the ratio of mirror ANCs, derived in Ref. 5], and discuss their validity. In Sec. III we briefly describe our microscopic cluster model and the ANCs associated with it. The results obtained in microscopic calculations are discussed in Sec. IV. A summary and conclusions are presented in Sec. V.

\section{OVERLAP INTEGRALS AND ANCS FOR MIRROR VIRTUAL DECAYS}

The $\mathrm{ANC} C_{l j}$ for the one-nucleon virtual decay $A \rightarrow$ $B+N$, where $B=A-1$, is defined via the tail of the overlap integral $I_{l j}(r)$

$$
I_{l j}(r)=\left\langle\chi_{\frac{1}{2} \tau}\left[\left[Y_{l}(\hat{\boldsymbol{r}}) \otimes \chi_{\frac{1}{2}}\right]_{j} \otimes \Psi^{J_{B}}\right]_{J_{A}} \mid \Psi^{J_{A}}\right\rangle
$$

between the many-body wave functions $\Psi^{J_{A}}$ and $\Psi^{J_{B}}$ of nuclei $A$ and $B$. Here $l$ is the orbital momentum, $j$ is the total relative angular momentum between $B$ and $N$, $\tau$ is the isospin projection and $\chi_{\frac{1}{2} \tau}$ is the isospin wave function of nucleon $N$, and $r$ is the distance between $N$ and the center-of-mass of $B$. Asymptotically, this overlap behaves as

$$
\sqrt{A} I_{l j}(r) \approx C_{l j} \frac{W_{-\eta, l+1 / 2}(2 \kappa r)}{r}, \quad r \rightarrow \infty,
$$

where $\kappa=\left(2 \mu \epsilon / \hbar^{2}\right)^{1 / 2}, \epsilon$ is the one-nucleon separation energy, $\eta=Z_{B} Z_{N} e^{2} \mu / \hbar^{2} \kappa, \mu$ is the reduced mass for the $B+N$ system and $W$ is the Whittaker function. According to Ref. [6], the ANC $C_{l j}$, multiplied by the trivial factor $i^{l} \pi^{\frac{1}{2}}(\hbar / \mu c)$, is equal to the on-shell amplitude (or vertex constant) of the one-nucleon virtual decay $A \rightarrow B+N$. This vertex constant can be written as a matrix element that contains the many-body wave functions of the nuclei $A$ and $B$. Therefore, the ANC $C_{l j}$ can also be represented by the same matrix element as follows [2, 7] 39] (4) is:

$$
\begin{aligned}
C_{l j} & =-\frac{2 \mu \sqrt{A}}{\hbar^{2}} \\
& \times\left\langle\chi_{\frac{1}{2} \tau}\left[\left[\varphi_{l}(i \kappa r) Y_{l}(\hat{\boldsymbol{r}}) \otimes \chi_{\frac{1}{2}}\right]_{j} \otimes \Psi^{J_{B}}\right]_{J_{A}}\|\hat{\mathcal{V}}\| \Psi^{J_{A}}\right\rangle(3)
\end{aligned}
$$

where

$$
\varphi_{l}(i \kappa r)=e^{-\frac{\pi i}{2}(l+1+\eta)} F_{l}(i \kappa r) / \kappa r
$$

$F_{l}$ is the regular Coulomb wave function at imaginary momentum $i \kappa$, and

$$
\hat{\mathcal{V}}=\sum_{i=1}^{B} V_{N N}\left(\left|\boldsymbol{r}_{i}-\boldsymbol{r}_{A}\right|\right)+\Delta V_{\text {Coul }}=\hat{\mathcal{V}}_{N}+\Delta V_{\text {Coul }}
$$

$$
\Delta V_{\text {Coul }}=\sum_{i=1}^{B} \frac{e_{i} e_{A}}{\left|\boldsymbol{r}_{i}-\boldsymbol{r}_{A}\right|}-\frac{Z_{B} e_{A} e}{r}
$$

Here $e_{i}\left(e_{A}\right)$ is the charge of the $i$-th $\left(A\right.$-th) nucleon, $Z_{B}$ is the charge of the residual nucleus $B$ and $V_{N N}$ is the twobody nuclear NN potential. If the separated nucleon is a neutron, $\varphi_{l}(i \kappa r)=i^{-l} j_{l}(i \kappa r)$ and $j_{l}(i \kappa r)$ is the spherical Bessel function.

It has been shown in Ref. [5] that the ratio

$$
\mathcal{R}=\left(\frac{C_{p}}{C_{n}}\right)^{2}
$$

where $C_{p}$ and $C_{n}$ are proton and neutron ANCs for mirror nucleon decays, can be approximated as follows:

$$
\mathcal{R} \approx \mathcal{R}_{0} \equiv\left|\frac{F_{l}\left(i \kappa_{p} R_{N}\right)}{\kappa_{p} R_{N} j_{l}\left(i \kappa_{n} R_{N}\right)}\right|^{2}
$$

Here $\kappa_{p}$ and $\kappa_{n}$ are determined by the proton and neutron separation energies $\epsilon_{p}$ and $\epsilon_{n}$ and $R_{N}$ is the radius of the nuclear interior to the choice of which the ratio $\mathcal{R}_{0}$ is not strongly sensitive.

The approximation (8) has been derived in Ref. [5] using Eq. (3) for mirror decays and assuming that 1) non-monopole on $r$ contributions from $\Delta V_{C o u l}$ are negligible; 2) differences in mirror wave functions inside the nuclear interior due to Coulomb interaction are not important and 3) charge symmetry of strong interactions is valid. As has been mentioned in Ref. [5], non-monopole contributions from $\Delta V_{\text {Coul }}$ increase the ratio $\mathcal{R}$. On the other hand, due to the stronger Coulomb interactions in $Z>N$ nuclei the magnitude of their wave functions are smaller in the nuclear interior as compared to the wave functions of $Z<N$ nuclei. This should lead to decrease of $\mathcal{R}$, which may become more noticeable for very small proton separation energy. Besides, if any nodes are present in the overlap $I_{l j}(r)$ then the contributions from $r>R_{N}$ to $C_{l j}$, determined by Eq. (3), may become larger. This can introduce further uncertainties into approximation (8) because differences in mirror proton and neutron wave functions in the $r>R_{N}$ region are important due to the Coulomb effects. It is possible, however, that all different factors may compensate each other so that, finally, the approximation (8) could be accurate enough to be used in practical purposes in the absence of more advanced detailed calculations.

Another approximation for $\mathcal{R}$ can be obtained if the overlap integral $I_{l j}(r)$ is thought of as being a normalised single-particle wave function times the spectroscopic factor $S$. In this case $C_{p(n)}=\sqrt{S_{p(n)}} b_{p(n)}$, where $b_{p(n)}$ is the single-particle proton (neutron) ANC. If charge symmetry is assumed both for the mirror single-particle wells and the mirror spectroscopic factors, then the ratio $\mathcal{R}$ is equal to the single-particle ratio

$$
\mathcal{R} \approx \mathcal{R}_{\text {s.p. }} \equiv\left(b_{p}^{\text {c.s. }} / b_{n}^{\text {c.s. }}\right)^{2},
$$

where $b_{p}^{\text {c.s. }}$ and $b_{n}^{\text {c.s. }}$ are calculated for exactly the same nuclear potential well. The accuracy of the approximation (9) is determined by the following factors: (i) the 
two-body potential model does not include effects of longrange contributions from non-monopole terms in $\Delta V_{\text {Coul }}$; (ii) the single particle potential wells for mirror pairs may differ because of slightly different matter distributions in their cores and (iii) the spectroscopic factors for mirror pairs may be not exactly the same.

Below, to understand better the validity of these approximations, we perform calculations of $\mathcal{R}$ for some light nuclei based on a microscopic cluster model.

\section{ONE-NUCLEON ANCS IN A MICROSCOPIC CLUSTER MODEL}

The cluster wave function for a nucleus $A$ consisting of a core $B$ and a nucleon $N$ can be represented as follows:

$$
\left.\Psi^{J_{A} M_{A}}=\sum_{l S J_{B} \omega} \mathcal{A}\left[\chi_{\frac{1}{2} \tau}\left[g_{\omega l S}^{J_{B}}(\boldsymbol{r}) \otimes\left[\Psi_{\omega}^{J_{B}} \otimes \chi_{\frac{1}{2}}\right]_{S}\right]_{J_{A} M_{A}}\right\rceil 10\right)
$$

where $\mathcal{A}=A^{-\frac{1}{2}}\left(1-\sum_{i=1}^{A-1} P_{i, A}\right)$ and the operator $P_{i, A}$ permutes spatial and spin-isospin coordinates of the $i$-th and $A$-th nucleons. In this work, $\Psi_{\omega}^{J_{B}}$ is a wave function of nucleus $B$ with the angular momentum $J_{B}$ defined either in translation-invariant harmonic-oscillator shell model, either in a multicluster model. The quantum number $\omega$ labels states with the same angular momentum $J_{B}$ and $S$ is the channel spin. The relative wave function $g_{\omega l S}^{J_{B}}(\boldsymbol{r})=g_{\omega l S}^{J_{B}}(r) Y_{l m}(\hat{r})$ also depends on $J_{B}$ and is determined from the solution of the Schrödinger equation for $\Psi^{J_{A} M_{A}}$ with some chosen NN potential. Below, we skip $J_{B}$ and $\omega$ in relative functions, overlap integrals and their characteristics for simplicity of notations.

The main advantage of a microscopic cluster model (MCM) is that it is able to provide the correct asymptotic behaviour for the overlap integral between $A$ and $B$. At large distances, $r \rightarrow \infty$, where the antisymmetrization between the external nucleon and the core is negligible, this overlap behaves as

$$
I_{l S}(r) \approx A^{-\frac{1}{2}} g_{l S}(r) \approx A^{-\frac{1}{2}} C_{l S} \frac{W_{-\eta, l+1 / 2}(2 \kappa r)}{r} .
$$

We achieve this type of behaviour by using the microscopic R-matrix approach [8] and determine the ANC $C_{l s}$ from the asymptotic behaviour of the relative wave functions corresponding to the $\omega$ state components $[9]$.

The MCM has been formulated in the $l S$ coupling scheme and the transition to the $l j$ coupling scheme is given by the standard transformation

$$
C_{l j}=\sum_{S}(-)^{J_{B}+\frac{1}{2}-S} \hat{S} \hat{j} W\left(J_{B} \frac{1}{2} J_{A} l ; S j\right) C_{l S},
$$

where $W$ is the Racah coefficient and $\hat{x}=(2 x+1)^{1 / 2}$. The same transformation is applicable to overlap integrals $I_{l j}$ and $I_{l S}$.

The MCM should provide more reliable ratios $\mathcal{R}$ for mirror ANCs than the approximations (8) and (9). Indeed, unlike in Eq. (8), the differences in the internal structure of mirror nuclei due to the Coulomb interaction are taken into account in the MCM. Also, determining the ANC directly from the tail of the overlap means that all the non-monopole contributions from $\Delta V_{C o u l}$ are present in the proton ANCs. The effects of core excitations are included as well. On the other hand, the MCM does not appeal to the concept of single-particle structure of nuclei and it does not need the hypothesis about charge symmetry for mirror single-particle potential wells and mirror spectroscopic factors. Charge symmetry for these quantities can still be studied within the MCM by investigating mirror spectroscopic factors, defined as norms of the MCM overlap integrals:

$$
S_{l j}=A \int_{0}^{\infty} d r r^{2}\left(I_{l j}(r)\right)^{2},
$$

and the single-particle ANCs $b_{l j}=C_{l j} S_{l j}^{-1 / 2}$. The latter is possible because the overlap integrals $I_{l j}(r)$, divided by the square root of their spectroscopic factors $S_{l j}$, are normalised functions of only one degree of freedom and they play the same role as single-particle wave functions generated by some effective local single-particle potential. Comparison between single-particle ANCs $b_{l j}$ for mirror nuclei may help to understand if mirror symmetry of the effective local single-particle potential wells is valid.

\section{RATIO OF MIRROR ANCS IN THE MCM}

\section{A. Mirror ANCs with charge independent NN interactions}

First of all, we have calculated ANCs for several nuclei assuming that $\mathrm{NN}$ interactions in mirror states are exactly the same. This assumption does not allow us to simultaneously reproduce the experimental neutron and proton separation energies in mirror states. However, it will enables us to explore the validity of the approximations (8) and (9). The effective NN interactions, used in this work, are the Volkov potential V2 [10] and the Minnesota (MN) potential [1]. The two-body spin-orbit force [12] and the Coulomb interaction are also included.

In this section, we have considered three mirror pairs: ${ }^{8} \mathrm{~B}\left(2^{+}\right)-{ }^{8} \mathrm{Li}\left(2^{+}\right),{ }^{13} \mathrm{~N}\left(\frac{1}{2}^{-}\right)-{ }^{13} \mathrm{C}\left(\frac{1}{2}^{-}\right)$and ${ }^{17} \mathrm{~F}\left(\frac{1}{2}^{+}\right)-{ }^{17} \mathrm{O}\left(\frac{1}{2}^{+}\right)$, which have been previously studied in Refs. 9, 13, 14, 15] in the $\alpha+{ }^{3} \mathrm{He}+\mathrm{p}(\alpha+\mathrm{t}+\mathrm{n})$, ${ }^{12} \mathrm{C}+\mathrm{p}\left({ }^{12} \mathrm{C}+\mathrm{n}\right)$ and ${ }^{16} \mathrm{O}+\mathrm{p}\left({ }^{16} \mathrm{O}+\mathrm{n}\right)$ microscopic cluster models. We have calculated the ANCs for these mirror pairs for several values of the parameters $m$ and $u$ of the V2 and MN interactions chosen to provide a range of theoretical separation energies covering the experimental separation energies. For each value of $m$ and $u$ we have calculated the ratio $\mathcal{R}_{M C M}=\left(C_{p}^{M C M} / C_{n}^{M C M}\right)^{2}$, using theoretical separation energies, and compared it to the analytical value $\mathcal{R}_{0}$ and single-particle estomate $\mathcal{R}_{\text {s.p. }}$. given by Eqs. (8) and (9). The ratios $\mathcal{R}_{M C M} / \mathcal{R}_{0}$ and $\mathcal{R}_{M C M} / \mathcal{R}_{\text {s.p. }}$ are shown in Fig. 1 . 

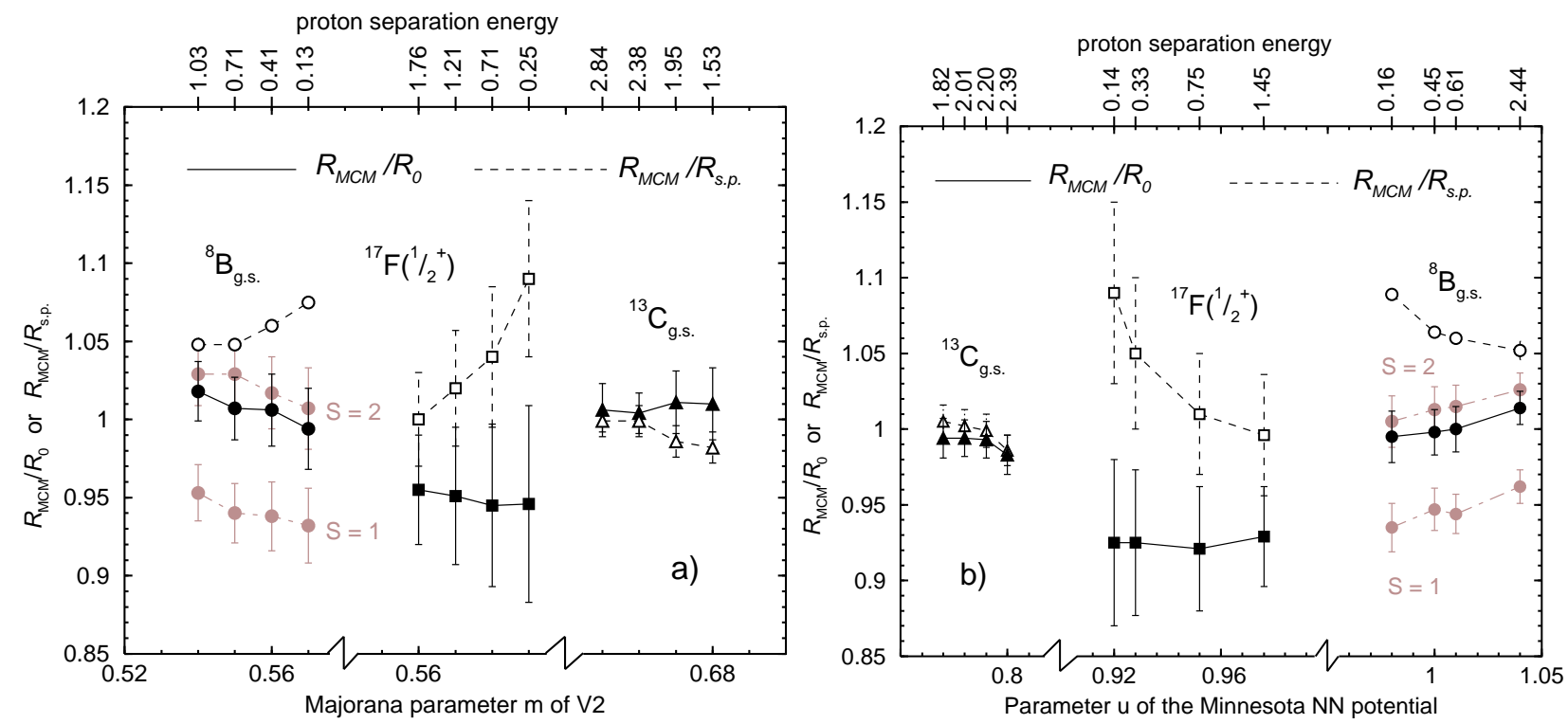

FIG. 1: Ratio $\mathcal{R}_{M C M} / \mathcal{R}_{0}$ (solid curves connecting black symbols) and $\mathcal{R}_{M C M} / \mathcal{R}_{\text {s.p. }}$ (dashed curves connecting open symbols) for different values of the Majorana parameter $m$ of the Volkov potential V2 $(a)$ and and for different parameter $u$ of the MN force $(b)$. The proton separation energies corresponding to each calculation are shown above upper horizontal axes. Grey symbols correspond to different channel spin $S$ in the ${ }^{8} \mathrm{~B}-{ }^{8} \mathrm{Li}$ mirror pair.

The error bars on Fig. 1 reflect the following uncertainties in the calculations of $\mathcal{R}_{0}$ and $\mathcal{R}_{\text {s.p. }}$. $\mathcal{R}_{0}$ depends on the range $R_{N}$ of the interaction potential between last nucleon $N$ and the core $B$. In Ref. [5] this range was taken as $1.3 B^{1 / 3}$. In fact, some contributions from the NN potential at larger $R_{N}$ may not be negligible, especially for cases when the wave function of the last nucleon has nodes. We have observed that, for all nuclei considered up to now, $\mathcal{R}_{0}$ slowly increases with $R_{N}$, reaches its maximum slightly beyond than $1.3 B^{1 / 3}$ and then slowly decreases. In estimating uncertainties in $\mathcal{R}_{0}$, we have assumed that its value is somewhere between $1.3 B^{1 / 3}$ and the maximum value. As for $\mathcal{R}_{\text {s.p. }}$, its uncertainties are due to the residual dependence on the nucleon-core potential. We have chosen this potential in the WoodsSaxon form and have varied its depth and radius at fixed diffusenesses to reproduce simultaneously the theoretical proton and neutron separation energies, calculated in the MCM. The uncertainties in $\mathcal{R}_{0}$ and $\mathcal{R}_{\text {s.p. }}$. vary with the choice of a mirror pair and are the largest for weakly-bound proton $s$-states with a node in their wave functions.

As shown in Fig.1, the precision of $\mathcal{R}_{0}$ and $\mathcal{R}_{\text {s.p. }}$ in approximating $\mathcal{R}_{M C M}$ varies for different systems. For the relatively strongly bound mirror pair ${ }^{13} \mathrm{~N}-{ }^{13} \mathrm{C}$, with the last nucleon in the $p$-wave with respect to the ${ }^{12} \mathrm{C}$ core, $\mathcal{R}_{M C M}$ agrees with $\mathcal{R}_{0}$ and $\mathcal{R}_{\text {s.p. }}$ within these uncertainties.

Another 0 p-shell mirror pair, ${ }^{8} \mathrm{~B}\left(2^{+}\right)-{ }^{8} \mathrm{Li}\left(2^{+}\right)$, is significantly less bound than ${ }^{13} \mathrm{~N}-{ }^{13} \mathrm{C}$. However, the quality of agreement between $\mathcal{R}_{M C M}$ and $\mathcal{R}_{0}$ for the ANCs squared summed over the channel spin, $C_{l}^{2}=C_{l S=1}^{2}+$
$C_{l S=2}^{2}$, is the same as in the ${ }^{13} \mathrm{~N}-{ }^{13} \mathrm{C}$ case (see black solid curves in Fig.1). In contrast, the single-particle estimate $\mathcal{R}_{\text {s.p. }}$ is larger than $\mathcal{R}_{M C M}$ and this difference increases with decreasing proton separation energy reaching $9 \%$. We recall that it is $C_{l}^{2}$ that determine the cross sections of the radiative capture reaction ${ }^{7} \mathrm{Be}(\mathrm{p}, \gamma)^{8} \mathrm{~B}$.

The ratios $\mathcal{R}_{M C M}$, calculated for spin channels $S=1$ and $S=2$, differ by $\sim 10 \%$ (see grey symbols in Fig.1). If the wave functions of mirror nuclei were exactly the same, then the ratio of mirror ANCs would not depend on the channel spin. The charge symmetry breaking due to the Coulomb interaction may manifest itself stronger in small components of the wave functions. Therefore, for such components, deviation from (8) can be more noticeable. Indeed, for ${ }^{8} \mathrm{~B}\left(2^{+}\right)-{ }^{8} \mathrm{Li}\left(2^{+}\right), C_{l S=2}^{2}$ are about four times larger than $C_{l S=1}^{2}$ and $\mathcal{R}_{M C M}$ for $S=2$ agrees with $\mathcal{R}_{0}$ better than in the channel with $S=1$.

In the last mirror pair considered in this section, ${ }^{17} \mathrm{~F}\left(\frac{1}{2}^{+}\right)-{ }^{17} \mathrm{O}\left(\frac{1}{2}^{+}\right)$, the valence proton and neutron are in $1 s$-state with respect to the core ${ }^{16} \mathrm{O}$. The calculated $\mathcal{R}_{M C M}$ values are about 5 to $8 \%$ smaller than $\mathcal{R}_{0}$ for all the proton separation energies considered. At the same time, $\mathcal{R}_{M C M}$ agrees with single-particle ratio $\mathcal{R}_{\text {s.p. }}$. if the the proton separation energy becomes larger than 1.4 MeV. When the NN interaction is changed so that the proton separation energy decrease down to $0.13 \mathrm{MeV}$ then $\mathcal{R}_{\text {s.p. }}$ overestimates $\mathcal{R}_{M C M}$ by about $9 \%$. 


\section{B. Mirror ANCs with charge symmetry breaking NN interactions}

Charge symmetry in realistic NN interactions is broken and this may be reflected in effective NN interactions. In the MCM calculations, different parameters $m$ and $u$ of the V2 and MN potentials should be taken in mirror states in order to achieve agreement between theoretical and experimental separation energies. A different choice of $m$ and $u$ in mirror states means that charge symmetry is still present in even $\mathrm{NN}$ interactions, but odd NN interactions are scaled with some renormalisation factor. We refer to this different choice as to charge symmetry breaking for the sake of simplicity, however, we do realise that it is not the same as for realistic NN potentials.

In this section, we calculate ANCs for several mirror pairs of nuclei that have two-, three- or four- cluster structure. In most cases, the wave functions of these nuclei have been obtained earlier. We calculate ANCs in the $l j$ coupling scheme as usually done in the analysis of transfer reactions, in which these ANCs can, or have been, determined. For nuclear astrophysics, the sum of the ANCs squared $C_{l}^{2}=C_{l j=1 / 2}^{2}+C_{l j=3 / 2}^{2}$ is often needed rather then their individual values in channels with different $j$. We show these values as well. Other characteristics of one-nucleon overlaps $\langle A \mid A-1\rangle$, namely, spectroscopic factors, r.m.s. radii and single-particle ANCs $b_{l j}^{2}=C_{l j}^{2} / S_{l j}$ are presented is this section as well.

$$
\text { 1. }{ }^{8} \mathrm{~B}-{ }^{8} \mathrm{Li}
$$

To reproduce experimental values of both the proton and neutron separation energies, the Majorana parameters $m$ of $\mathrm{V} 2$ should differ in ${ }^{8} \mathrm{~B}$ and ${ }^{8} \mathrm{Li}$ by $1.8 \%$. For the MN potential, this difference is only $1.0 \%$.

The $C_{1 \frac{3}{2}}^{2}$ values obtained with the $\mathrm{V} 2$ potential are by $22-26 \%$ larger than those calculated with MN (see Table I). However, the ratio $\mathcal{R}_{\frac{3}{2}}=C_{1 \frac{3}{2}}^{2}(p) / C_{1 \frac{3}{2}}^{2}(n)$ changes only by $3 \%$ with the NN potential choice. These ratios, 1.048 for $\mathrm{V} 2$ and 1.079 for $\mathrm{MN}$, are smaller than the value $\mathcal{R}_{0}=$ $1.13 \pm 0.01$ predicted by the formula (8) but higher than the single-particle value $\mathcal{R}_{\text {s.p. }}=1.01 \pm 0.01$ obtained from equality of mirror proton and neutron single-particle potential wells and the mirror proton and neutron spectroscopic factors.

The $C_{1 \frac{1}{2}}^{2}$ values are much smaller then $C_{1 \frac{3}{2}}^{2}$ and they change only by $9 \%$ for ${ }^{8} \mathrm{~B}$ and $4 \%$ for ${ }^{8} \mathrm{Li}$ with different NN potential choices. The ratio $\mathcal{R}_{\frac{1}{2}}=C_{1 \frac{1}{2}}^{2}(p) / C_{1 \frac{1}{2}}^{2}(n)$ of the mirror ANCs in this case, 1.26 and 1.19 for the V2 and MN potentials respectively, are by $20 \%$ and $10 \%$ larger than $\mathcal{R}_{\frac{3}{2}}$, which should be due to the stronger influence of charge symmetry breaking effects in the small $j=1 / 2$ component.

The $C_{l}^{2}$ value increases by $20 \%$ with a change of the $\mathrm{NN}$ force. However, the ratio $\mathcal{R}_{M C M}$ changes only within
2\%, being 1.068 and 1.092 for $\mathrm{V} 2$ and MN respectively. Its average value of 1.08 is closer to the analytical value $\mathcal{R}_{0}=1.13 \pm 0.01$ than to the single-particle value $\mathcal{R}_{\text {s.p. }}=$ $1.01 \pm 0.01$. We recall that for charge independent $\mathrm{NN}$ interactions, the difference in $\mathcal{R}_{M C M}$ and $\mathcal{R}_{0}$ is only about $2 \%$ for energies $\epsilon_{p}$ similar to the experimental ones.

The proton ANCs for ${ }^{8} \mathrm{~B}$ have been determined in Ref. [16] using the $\left({ }^{7} \mathrm{Be},{ }^{8} \mathrm{~B}\right)$ transfer reactions on two different targets, ${ }^{14} \mathrm{~N}$ and ${ }^{10} \mathrm{~B}$. The average value of $C_{1}^{2}$ deduced from these experiments is $0.449 \pm 0.045 \mathrm{fm}^{-1}$. The breakup reaction at intermediate energies gave a very close value of $0.450 \pm 0.039 \mathrm{fm}^{-1}$ 17]. These values are by $42 \%$ and $30 \%$ smaller than the MCM predictions with the $\mathrm{V} 2$ and $\mathrm{MN}$ potentials respectively. The neutron ANC of the mirror nucleus ${ }^{8} \mathrm{Li}$ has been experimentally determined in Ref. [18] using transfer reaction ${ }^{13} \mathrm{C}\left({ }^{7} \mathrm{Li},{ }^{8} \mathrm{Li}\right){ }^{12} \mathrm{C}$. Its value, $C_{1}^{2}=0.449 \pm 0.045 \mathrm{fm}^{-1}$, is also much smaller than the predictions of the MCM. However, the ratio of the experimentally determined mirror ANCs is $1.08 \pm 0.15$, which in excellent agreement with the average MCM ratio.

The shapes of the angular distributions of the transfer reaction ${ }^{13} \mathrm{C}\left({ }^{7} \mathrm{Li},{ }^{8} \mathrm{Li}\right){ }^{12} \mathrm{C}$ are very sensitive to the interference between the contributions from the overlap integrals with different $j$. It was found in Ref. 18 that $C_{1 \frac{3}{2}}^{2} / C_{1 \frac{1}{2}}^{2}=0.13(2)$. This value is in excellent agreement with the value of 0.131 obtained in the MCM using the MN potential. As for $\mathrm{V} 2$, it predicts for $C_{1 \frac{3}{2}}^{2} / C_{1 \frac{1}{2}}^{2}$ much lower ratio, equal to 0.108 .

The mirror spectroscopic factors $S_{1 \frac{3}{2}}$ of large components of the overlap integrals differ by about $1 \%$ and they change less than by $1 \%$ with different choice of the NN potential (see Table I). However, small spectroscopic factors $S_{1 \frac{1}{2}}$ are more sensitive to the NN potentials choice and their difference in mirror states reaches $20 \%$. The sums of mirror spectroscopic factors $S_{1}=S_{1 \frac{3}{2}}+S_{1 \frac{1}{2}}$ differ in mirror nuclei ${ }^{8} \mathrm{Li}$ and ${ }^{8} \mathrm{~B}$ only by $2 \%$.

The values $b_{l j}^{2}$ calculated with the V2 and MN potentials are presented in Table 1 as well. The ratio of $b_{l j}^{2}$ for the mirror overlaps differs by 5 to $10 \%$ from the singleparticle estimate $\mathcal{R}_{\text {s.p. }}=1.01 \pm 0.01$ obtained on the assumption that mirror single-particle potential wells are exactly the same. Therefore, the present MCM calculations suggest that this assumption is not valid.

It is interesting to note that MCM predicts that the r.m.s. radius $\left\langle r_{1 \frac{1}{2}}^{2}\right\rangle^{1 / 2}$ should be larger than $\left\langle r_{1 \frac{3}{2}}^{2}\right\rangle^{1 / 2}$. The same result has been obtained earlier in Ref. [2] where the overlap integrals were found as solutions of the inhomogeneous equation with a shell model source term. A standard single-particle potential model with central and spin-orbit potentials predicts that the singleparticle wave function with $j=3 / 2$ has smaller radius than the one with $j=1 / 2$. To achieve this inversion of the r.m.s. radii, the phenomenological single-particle spin-orbit potential should be taken with opposite sign. Understanding the differences in $j=3 / 2$ and $j=1 / 2$ 
overlaps is important for the accurate determination of ANC from transfer reactions.

$$
\text { 2. }{ }^{12} \mathrm{~B}-{ }^{12} \mathrm{~N}
$$

To study the overlap integrals $\left\langle{ }^{12} \mathrm{~N} \mid{ }^{11} \mathrm{C}\right\rangle$ and $\left\langle{ }^{12} \mathrm{~B} \mid{ }^{11} \mathrm{~B}\right\rangle$ we use the wave functions of ${ }^{12} \mathrm{~B}$ and ${ }^{12} \mathrm{~N}$ calculated earlier in Ref. [19] in the multichannel two-cluster models ${ }^{11} \mathrm{~B}+\mathrm{n}$ and ${ }^{11} \mathrm{C}+\mathrm{p}$ with excited states $\frac{1}{2}^{-}, \frac{3}{2}^{-}, \frac{5}{2}^{-}$and $\frac{7}{2}^{-}$of the ${ }^{11} \mathrm{~B}$ and ${ }^{11} \mathrm{C}$ cores taken into account.

The ANCs, spectroscopic factors, r.m.s. radii of these overlaps and the single-particle ANCs $b_{l j}$ are presented in Table I. The dependence of these values on the NN potential choice is weaker than in the case of ${ }^{8} \mathrm{Li}^{8}{ }^{8} \mathrm{~B}$. The ratio $\mathcal{R}_{M C M}$ depends on the NN potential choice less than the ANCs themselves, and the difference between $\mathcal{R}_{\frac{3}{2}}$ and $\mathcal{R}_{\frac{1}{2}}$ is smaller than for the ${ }^{8} \mathrm{~B}-{ }^{8} \mathrm{Li}$ mirror pair. $\mathcal{R}_{M C M}$, which is equal to 1.29 for $\mathrm{V} 2$ and 1.30 for $\mathrm{MN}$, agrees well with the single-particle estimate $\mathcal{R}_{\text {s.p. }}=1.30 \pm 0.02$ obtained on the assumption of charge-symmetry of mirror single-particle potential wells. However, it is smaller than the prediction $\mathcal{R}_{0}=1.38 \pm 0.02$ of Eq. (8) by $6 \%$. In section IV.A we have shown that for the $p$-shell nucleus ${ }^{8} \mathrm{~B}$ with the proton separation energy similar to that in ${ }^{12} \mathrm{~N}, \mathcal{R}_{M C M}$ agrees with $\mathcal{R}_{0}$ within uncertainties of the calculation of the latter (see $m=0.56$ and $u=1.01$ cases in Fig.1). Therefore, the $6 \%$ deviation of $\mathcal{R}_{M C M}$ from $\mathcal{R}_{0}$, obtained in this section, can be attributed to the charge symmetry breaking in the effective NN interactions, which is about $1.9 \%$ for $\mathrm{V} 2$ and $5.8 \%$ for $\mathrm{MN}$.

The neutron ANC $C_{l}^{e x p}=1.16 \pm 0.10 \mathrm{fm}^{-1 / 2}$ and the r.m.s. radius $\left\langle r_{\text {exp }}^{2}\right\rangle^{1 / 2}=3.16 \pm 0.32 \mathrm{fm}$ for $\left\langle\left.{ }^{12} \mathrm{~B}\right|^{11} \mathrm{~B}\right\rangle$ have been reported in Ref. [20] where they have been determined from the ${ }^{11} \mathrm{~B}(\mathrm{~d}, \mathrm{p}){ }^{12} \mathrm{~B}$ reaction. Our MCM calculations give the larger values, $C_{l}=1.35 \mathrm{fm}^{-1 / 2}$ for V2 and and $C_{l}=1.28 \mathrm{fm}^{-1 / 2}$ for MN, while the theoretical r.m.s. radius ranges from 3.41 to $3.59 \mathrm{fm}$ depending on $j$ and $\mathrm{NN}$ force.

The proton ANCs for ${ }^{12} \mathrm{~N}$ have been determined from the peripheral transfer reaction ${ }^{14} \mathrm{~N}\left({ }^{11} \mathrm{C},{ }^{12} \mathrm{~N}\right){ }^{13} \mathrm{C}$ in Ref. 21] resulting in $C_{l \frac{1}{2}}^{2}=1.4 \pm 0.2 \mathrm{fm}^{-1 / 2}, C_{l \frac{3}{2}}^{2}=0.33 \pm$ $0.05 \mathrm{fm}^{-1 / 2}$ and $C_{l}^{2}=C_{l \frac{1}{2}}^{2}+C_{l \frac{3}{2}}^{2}=1.73 \pm 0.25 \mathrm{fm}^{-1 / 2}$. Our MCM calculations overestimate the experimental $C_{l}^{2}$ value by $35 \%$ for $\mathrm{V} 2$ and $23 \%$ for MN. The theoretical ratio $C_{l \frac{3}{2}}^{2} / C_{l \frac{1}{2}}^{2} 0.34$ for $\mathrm{V} 2$ and 0.39 for $\mathrm{MN}$, is also larger than the experimental value of $0.24 \pm 0.07$. However, the ANCs in mirror nuclei are overestimated in the same proportion, so that the theoretical ratio $\mathcal{R}_{M C M}$ of 1.29 and 1.30 agrees well with the experimental value $\mathcal{R}_{\text {exp }}=$ $1.28 \pm 0.29$.

The spectroscopic factors in ${ }^{12} \mathrm{~N}$ and ${ }^{12} \mathrm{~B}$ change by no more than $6 \%$ with different choices of the NN potential. The mirror spectroscopic factors $S_{1 \frac{1}{2}}$, differ by $2.8 \%$ and $3.6 \%$ for $\mathrm{V} 2$ and MN respectively while $S_{1 \frac{1}{2}}$ are practically the same for both of them.
3. ${ }^{13} \mathrm{C}-{ }^{13} \mathrm{~N}$

To describe the mirror pair ${ }^{13} \mathrm{~N}-{ }^{13} \mathrm{C}$, we used two different models: the multichannel two-cluster model ${ }^{12} \mathrm{C}$ $+\mathrm{n}(\mathrm{p})$ from Ref. [15] and the multichannel four-cluster model $\alpha+\alpha+\alpha+\mathrm{n}(\mathrm{p})$, that has been developed in Ref. 22. Numerical precision of ANCs squared obtained in the latter model is about 2-3\%. The results of calculations are presented in Table I.

The ANCs obtained in two-cluster and four-cluster models differ by 60 to $80 \%$ and the spectroscopic factors differ by about 50 to $60 \%$ depending on the NN potential used in calculations. Such a large difference arises because the $\alpha+\alpha+\alpha$ model for the nucleus ${ }^{12} \mathrm{C}$ contains only one type of permutational symmetry determined by the Young diagram $[f]=[444]$. As explained in Ref. [15], the main contribution to the spectroscopic factor, vertex constant, and therefore, to the ANC of the overlap integral $\left\langle{ }^{13} \mathrm{C} \mid{ }^{12} \mathrm{C}\right\rangle$ comes from the overlap between the $[4441]{ }^{22} \mathrm{P}$ state in ${ }^{13} \mathrm{C}$ and the $[4431]{ }^{13} \mathrm{P}$ state in ${ }^{12} \mathrm{C}$. The $[4431]^{13} \mathrm{P}$ configuration is absent in the $\alpha+\alpha+\alpha$ model but is present in the one-center shell model wave function of ${ }^{12} \mathrm{C}$ used in the two-cluster model. For this reason, the two-cluster model gives larger ANCs, and spectroscopic factors for ${ }^{13} \mathrm{C}$ and ${ }^{13} \mathrm{~N}$, than the four-cluster model.

Several experimental values for the neutron ANC of ${ }^{13} \mathrm{C}$ are available 20, 23, 24, 25, 26, 27]. Apart from the latest value from Ref. [20], obtained from a nonperipheral $(\mathrm{d}, \mathrm{p})$ reaction, they agree with each other leading to an average value $C_{l}^{2}=2.36 \pm 0.12 \mathrm{fm}^{-1}$. Our two-cluster calculations with V2 agree with this value while the same calculations performed with MN underestimate it. However, such calculations are very sensitive to the spin-orbit force, as it regulates the probability of the $[4431]{ }^{13} \mathrm{P}$ configuration in ${ }^{12} \mathrm{C}[15]$. As for the fourbody model, it underestimates the experimental values $C_{l}^{2}$ squared by a factor of two.

The ratio $\mathcal{R}_{M C M}$, calculated in the four-cluster model agrees well both with the analytical value $\mathcal{R}_{0}=1.198 \pm$ 0.004 and the single-particle value $\mathcal{R}_{\text {s.p. }}=1.168 \pm 0.020$. However, the two-cluster model gives smaller values of $\mathcal{R}_{M C M}, 1.13$ and 1.14 for the V2 and MN potentials respectively. As we have seen in Sec. IV.A, the two-cluster model $\mathcal{R}_{M C M}$ agrees both with $\mathcal{R}_{0}$ and $\mathcal{R}_{\text {s.p. }}$ if the charge symmetry of the NN interactions is present. In this section, to reproduce the mirror separation energies $\epsilon_{p}$ and $\epsilon_{n}$ within the two-cluster model, the Majorana parameters $m$ of V2 in mirror nuclei ${ }^{13} \mathrm{C}$ and ${ }^{13} \mathrm{~N}$ have to be different by $1.4 \%$ and the parameters $u$ of MN must differ by $1.9 \%$, which corresponds to $\sim 6 \%$ difference in the odd NN potentials. With actual parameters $m$ and $u$, used in the two-cluster calculations, the singlet- and triplet-odd parts of the NN potentials are large. As a result, the deviation of $\mathcal{R}_{M C M}$ from what would be expected in the case of charge symmetry, is comparable to the degree in which charge symmetry is broken. The situation is different for the four-cluster model where the required charge symmetry breaking in these components is also smaller 
TABLE I: Asymptotic normalization coefficients squared $C_{l j}^{2}$ (in $\mathrm{fm}^{-1}$ ), their sums $C_{l}^{2}$, spectroscopic factors $S_{l j}$ and $S_{l}=$ $S_{l, l-\frac{1}{2}}+S_{l, l+\frac{1}{2}}$, ratio $b_{l j}^{2}=C_{l j}^{2} / S_{l j}\left(\right.$ in $\mathrm{fm}^{-1}$ ) and r.m.s. radii $\left\langle r_{l j}^{2}\right\rangle^{1 / 2}$ (in $\mathrm{fm}$ ) for mirror overlap integrals. The calculations have been performed with two NN potentials, V2 and MN. The ratios $\mathcal{R}$ of similar quantities of mirror overlaps are given in each third line.

\begin{tabular}{|c|c|c|c|c|c|c|c|c|c|c|c|c|}
\hline & & $l$ & $C_{l, l-\frac{1}{2}}^{2}$ & $C_{l, l+\frac{1}{2}}^{2}$ & $C_{l}^{2}$ & $S_{l, l-\frac{1}{2}}$ & $S_{l, l+\frac{1}{2}}$ & $S_{l}$ & $b_{l, l-\frac{1}{2}}^{2}$ & $b_{l, l+\frac{1}{2}}^{2}$ & $\left\langle r_{l, l-\frac{1}{2}}^{2}\right\rangle^{1 / 2}$ & $\left\langle r_{l, l+\frac{1}{2}}^{2}\right\rangle^{1 / 2}$ \\
\hline \multirow{4}{*}{ V2 } & & & & & $\left.3\left(2^{+}\right)\right|^{7}$ & $-)\rangle-\left\langle^{8}\right.$ & $\left.2^{+}\right)\left.\right|^{7} \mathrm{Li}\left(\frac{3}{2}^{-}\right.$ & $\overline{)\rangle}$ & & & & \\
\hline & $p$ & 1 & 0.0886 & 0.6850 & 0.7736 & 0.104 & 0.926 & 1.030 & 0.850 & 0.740 & 5.08 & 4.83 \\
\hline & $n$ & 1 & 0.0706 & 0.6539 & 0.7244 & 0.086 & 0.955 & 1.040 & 0.823 & 0.685 & 4.078 & 3.87 \\
\hline & $\mathcal{R}$ & & 1.256 & 1.048 & 1.068 & 1.217 & 0.970 & 0.990 & 1.033 & 1.098 & & \\
\hline \multirow{3}{*}{ MN } & $p$ & 1 & 0.0811 & 0.5602 & 0.6413 & 0.114 & 0.922 & 1.037 & 0.709 & 0.607 & 4.76 & 4.51 \\
\hline & $n$ & 1 & 0.0682 & 0.5193 & 0.5875 & 0.102 & 0.942 & 1.044 & 0.668 & 0.552 & 3.83 & 3.64 \\
\hline & $\mathcal{R}$ & & 1.189 & 1.079 & 1.092 & 1.119 & 0.979 & 0.993 & 1.06 & 1.10 & & \\
\hline \multicolumn{13}{|c|}{$\left\langle\left.{ }^{12} \mathrm{~N}\left(1^{+}\right)\right|^{11} \mathrm{C}\left(\frac{3}{2}^{-}\right)\right\rangle-\left\langle\left.{ }^{12} \mathrm{~B}\left(1^{+}\right)\right|^{11} \mathrm{~B}\left(\frac{3}{2}^{-}\right)\right\rangle$} \\
\hline \multirow{3}{*}{ V2 } & $p$ & 1 & 1.76 & 0.595 & 2.35 & 0.672 & 0.261 & 0.933 & 2.61 & 2.28 & 4.07 & 3.92 \\
\hline & $n$ & 1 & 1.38 & 0.445 & 1.82 & 0.684 & 0.262 & 0.946 & 2.02 & 1.70 & 3.59 & 3.47 \\
\hline & $\mathcal{R}$ & & 1.28 & 1.34 & 1.29 & 0.982 & 0.996 & 0.986 & 1.29 & 1.34 & & \\
\hline \multirow{3}{*}{ MN } & $p$ & 1 & 1.529 & 0.598 & 2.127 & 0.637 & 0.276 & 0.913 & 2.40 & 2.17 & 3.97 & 3.86 \\
\hline & $n$ & 1 & 1.201 & 0.440 & 1.641 & 0.661 & 0.278 & 0.939 & 1.82 & 1.58 & 3.50 & 3.41 \\
\hline & $\mathcal{R}$ & & 1.27 & 1.36 & 1.30 & 0.964 & 0.993 & 0.972 & 1.32 & 1.37 & & \\
\hline \multicolumn{13}{|c|}{$\overline{\left\langle/{ }^{13} \mathrm{~N}\left(\frac{1}{2}^{-}\right) \mid{ }^{12} \mathrm{C}\left(0^{+}\right)\right\rangle-\left\langle{ }^{13} \mathrm{C}\left(\frac{1}{2}^{-}\right) \mid{ }^{12} \mathrm{C}\left(0^{+}\right)\right\rangle \text {two-cluster model }}$} \\
\hline \multirow{3}{*}{ V2 } & $p$ & 1 & & 2.66 & & & 0.530 & & & 5.01 & & 3.63 \\
\hline & $n$ & 1 & & 2.36 & & & 0.531 & & & 4.45 & & 3.37 \\
\hline & $\mathcal{R}$ & & & 1.13 & & & 0.998 & & & 1.13 & & \\
\hline & $p$ & 1 & & 2.18 & & & 0.502 & & & 4.35 & & 3.50 \\
\hline $\mathrm{MN}$ & $n$ & 1 & & 1.92 & & & 0.498 & & & 3.85 & & 3.26 \\
\hline & $\mathcal{R}$ & & & 1.14 & & & 1.008 & & & 1.13 & & \\
\hline & & & & $\left\langle^{13} \mathrm{~N}\left(\frac{1}{2}^{-}\right)\right.$ & $\overline{\left.\mathrm{C}\left(0^{+}\right)\right\rangle-}$ & $\overline{\left.\mathrm{C}\left(\frac{1}{2}^{-}\right)\right|^{1}}$ & $\left.\left(0^{+}\right)\right\rangle$four-c & luster $\mathrm{n}$ & & & & \\
\hline & $p$ & 1 & & $1.54 \pm 0.04$ & & & 0.335 & & & $4.61 \pm 0.10$ & & 3.58 \\
\hline V2 & $n$ & 1 & & $1.30 \pm 0.04$ & & & 0.330 & & & $3.95 \pm 0.12$ & & 3.32 \\
\hline & $\mathcal{R}$ & & & $1.19 \pm 0.01$ & & & 1.01 & & & $1.17 \pm 0.01$ & & \\
\hline & $p$ & 1 & & $1.34 \pm 0.04$ & & & 0.341 & & & $3.93 \pm 0.12$ & & 3.44 \\
\hline MN & $n$ & 1 & & $1.12 \pm 0.06$ & & & 0.336 & & & $3.33 \pm 0.18$ & & 3.20 \\
\hline & $\mathcal{R}$ & & & $1.19 \pm 0.01$ & & & 1.01 & & & $1.17 \pm 0.01$ & & \\
\hline & & & & 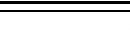 & $\overline{\overline{\left.\left(\frac{1}{2}^{-}\right)\right|^{1}}}$ & $\overline{\overline{+})\rangle-\left\langle{ }^{1}\right.}$ & $\overline{\left.\left(^{\frac{1}{2}}{ }^{-}\right)\right|^{14} \mathrm{~N}(1}$ & & & & & \\
\hline & $p$ & 1 & 64.7 & 0.830 & 65.5 & 1.420 & 0.017 & 1.437 & 45.6 & 48.8 & 3.10 & 3.15 \\
\hline V2 & $n$ & 1 & 43.9 & 0.568 & 44.5 & 1.456 & 0.017 & 1.473 & 30.2 & 33.27 & 3.00 & 3.05 \\
\hline & $\mathcal{R}$ & & 1.473 & 1.461 & 1.473 & 0.975 & 1.006 & 0.976 & 1.511 & 1.465 & & \\
\hline & $p$ & 1 & 52.7 & 0.051 & 52.7 & 1.465 & $8.6 \times 10^{-4}$ & 1.466 & 35.9 & 58.6 & 2.98 & 3.35 \\
\hline MN & $n$ & 1 & 35.6 & 0.036 & 35.6 & 1.489 & $8.2 \times 10^{-4}$ & 1.489 & 23.9 & 43.7 & 2.89 & 3.29 \\
\hline & $\mathcal{R}$ & & 1.479 & 1.417 & 1.481 & 0.984 & 1.049 & 0.985 & 1.502 & 1.34 & & \\
\hline & & & & - & $\overline{\left.\left(\frac{3}{2}^{+}\right)\right|^{1}}$ & $\left.\left.{ }^{+}\right)\right\rangle-\left\langle{ }^{1}\right.$ & $\left.\left(\frac{3}{2}^{+}\right)\right|^{14} \mathrm{~N}(1$ & & & & & \\
\hline & $p$ & 0 & & 33.18 & & & 0.986 & & & 33.7 & & 5.01 \\
\hline V2 & $n$ & 0 & & 8.74 & & & 0.953 & & & 9.17 & & 4.15 \\
\hline & $\mathcal{R}$ & & & 3.79 & & & 1.035 & & & 3.68 & & \\
\hline & $p$ & 0 & & 29.4 & & & 0.995 & & & 29.6 & & 4.80 \\
\hline MN & $n$ & 0 & & 7.67 & & & 0.966 & & & 7.93 & & 3.99 \\
\hline & $\mathcal{R}$ & & & 3.82 & & & 1.03 & & & 3.73 & & \\
\hline & & & & 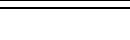 & $\overline{\left.\left(\frac{5}{2}+\right)\right|^{1}}$ & $\overline{\left.\left.{ }^{+}\right)\right\rangle-\left\langle^{1}\right.}$ & $\left.\frac{5}{2}^{+}\right)\left.\right|^{16} \mathrm{O}(0$ & & & & & \\
\hline & $p$ & 2 & & 1.09 & & & 1.122 & & & 1.056 & & 3.84 \\
\hline V2 & $n$ & 2 & & 1.00 & & & 1.125 & & & 0.889 & & 3.61 \\
\hline & $\mathcal{R}$ & & & 1.19 & & & 0.997 & & & 1.19 & & \\
\hline & $p$ & 2 & & 0.951 & & & 1.124 & & & 0.846 & & 3.67 \\
\hline $\mathrm{MN}$ & $n$ & 2 & & 0.796 & & & 1.126 & & & 0.706 & & 3.47 \\
\hline & $\mathcal{R}$ & & & 1.19 & & & 0.998 & & & 1.20 & & \\
\hline & & & & (2) & $\overline{\left.\left(\frac{1}{2}^{+}\right)\right|^{1}}$ & $\overline{\left.\left.{ }^{+}\right)\right\rangle-\left\langle^{1}\right.}$ & $\left.\left(\frac{1{ }^{+}}{2}\right)\right|^{16} \mathrm{O}(0$ & & & & & \\
\hline & $p$ & 0 & & 8000 & & & 1.095 & & & 7277 & & 5.55 \\
\hline V2 & $n$ & 0 & & 11.0 & & & 1.110 & & & 9.93 & & 4.40 \\
\hline & $\mathcal{R}$ & & & 727 & & & 0.986 & & & 733 & & \\
\hline & $p$ & 0 & & 7110 & & & 1.110 & & & 6444 & & 5.32 \\
\hline $\mathrm{MN}$ & $n$ & 0 & & 9.66 & & & 1.113 & & & 8.68 & & 4.24 \\
\hline & $\mathcal{R}$ & & & 736 & & & 0.997 & & & 742 & & \\
\hline & & & & & $\left.\frac{5^{+}}{2}\right)\left.\right|^{22}$ & $\left.\left.{ }^{+}\right)\right\rangle-\left\langle^{2}\right.$ & $\left.\left(\frac{5}{2}^{+}\right)\right|^{22} \mathrm{Ne}$ & $\overline{\left.\left(0^{+}\right)\right\rangle}$ & & & & \\
\hline & $p$ & 2 & & $1.17 \times 10^{4}$ & & & 0.285 & & & $4.12 \times 10^{4}$ & & 3.93 \\
\hline V2 & $n$ & 2 & & 0.398 & & & 0.299 & & & 1.33 & & 3.68 \\
\hline & $\mathcal{R}$ & & & $2.95 \times 10^{4}$ & & & 0.953 & & & $3.11 \times 10^{4}$ & & \\
\hline & $p$ & 2 & & $1.02 \times 10^{4}$ & & & 0.281 & & & $3.61 \times 10^{4}$ & & 3.83 \\
\hline $\mathrm{MN}$ & $n$ & 2 & & 0.343 & & & 0.294 & & & 1.17 & & 3.60 \\
\hline & $\mathcal{R}$ & & & $2.96 \times 10^{4}$ & & & 0.956 & & & $3.09 \times 10^{4}$ & & \\
\hline & & & & & $\overline{\left.\frac{1}{2}^{+}\right)\left.\right|^{26} \mathrm{~s}}$ & )$\rangle-\left\langle{ }^{27}\right.$ & $\left.\left(\frac{1}{2}{ }^{+}\right)\right|^{26} \mathrm{Mg}($ & $\overline{\overline{\left.\left.0^{+}\right)\right\rangle}}$ & & & & \\
\hline & $p$ & 0 & & 1648 & & & 0.901 & & & 1830 & & 4.43 \\
\hline V2 & $n$ & 0 & & 36.0 & & & 0.824 & & & 45.2 & & 3.93 \\
\hline & $\mathcal{R}$ & & & 45.8 & & & 1.09 & & & 40.5 & & \\
\hline & $p$ & 0 & & 1380 & & & 0.873 & & & 1582 & & 4.28 \\
\hline $\mathrm{MN}$ & $n$ & 0 & & 31.1 & & & 0.809 & & & 38.5 & & 3.81 \\
\hline & $\mathcal{R}$ & & & 44.3 & & & 1.08 & & & 41.1 & & \\
\hline
\end{tabular}


and the actual choice of parameters $m$ and $u$ gives to weaker odd NN potentials.

The spectroscopic factors obtained are sensitive both to the model and the NN potential choice, however the difference in mirror spectroscopic factors does not exceed $2 \%$. The same model that reproduces the experimental ANC value in ${ }^{13} \mathrm{C}$ gives the spectroscopic factor $S=0.53$ which is lower than the shell model value of 0.68 of Ref. [28].

$$
\text { 4. }{ }^{15} \mathrm{O}-{ }^{15} \mathrm{~N}
$$

We describe ${ }^{15} \mathrm{O}$ and ${ }^{15} \mathrm{~N}$ in the multichannel twocluster ${ }^{14} \mathrm{~N}+\mathrm{p}(\mathrm{n})$ model with the core ${ }^{14} \mathrm{~N}$ being either in the ground state or in one of the first excited states $1^{+}, 2^{+}$or $3^{+}$. The internal structure of the ${ }^{14} \mathrm{~N}$ core is represented by the $0 p$ oscillator shell model with the oscillator radius of $1.6 \mathrm{fm}$. We consider only two states in ${ }^{15} \mathrm{O}$ and ${ }^{15} \mathrm{~N}$, the ground state and the first $3 / 2^{+}$state, since they are the most important for understanding ${ }^{15} \mathrm{O}$ production in the $\mathrm{CNO}$ cycle.

For the ground states of ${ }^{15} \mathrm{O}$ and ${ }^{15} \mathrm{~N},\left|C_{1 \frac{3}{2}}\right|^{2}$ are about two orders of magnitude smaller than $\left|C_{1 \frac{1}{2}}\right|^{2}$ for both NN potentials used in the calculations (see Table I), while the experimentally determined $\left|C_{1 \frac{3}{2}}\right|^{2}$ is only one tenth of $\left|C_{1 \frac{1}{2}}\right|^{2}$ [29]. The $\left|C_{l}\right|^{2}=65.5 \mathrm{fm}^{-1}$ value calculated with V2 agrees with experimental value of $63 \pm 14 \mathrm{fm}^{-1}$ from Ref. 30], while $\left|C_{l}\right|^{2}=52.7 \mathrm{fm}^{-1}$ calculated with MN agrees with another available experimental value of $54 \pm 5.9 \mathrm{fm}^{-1}$ [29]. The ratio $\mathcal{R}_{M C M}=1.48$, which is almost the same for both NN potentials, agrees well with the analytical value $\mathcal{R}_{0}=1.48$ obtained from Eq. (8) and with single-particle value $\mathcal{R}_{\text {s.p. }}=1.51 \pm 0.03$.

The difference between mirror spectroscopic factors does not exceed $2.5 \%$ for $j=1 / 2$, but it is slightly larger for $j=3 / 2$ and the MN potential. This difference is most likely due to the $\sim 3 \%$ difference in the NN potential parameters in mirror states required for simultaneous reproduction of proton and neutron separation energies in ${ }^{15} \mathrm{O}$ and ${ }^{15} \mathrm{~N}$.

The ANCs for the first excited $3 / 2^{+}$state is less sensitive to the NN potential choice than those for the ground state. An experimentally determined value $C_{l}^{2}=21 \pm$ $5 \mathrm{fm}^{-1}$ for the $\left\langle{ }^{15} \mathrm{O}\left(\frac{3}{2}_{1}^{+}\right) \mid{ }^{14} \mathrm{~N}\right\rangle$ has been reported in Ref. 30]. The experimental data from this work have been recently reanalysed in Ref. 29], increasing this value to $C_{l}^{2}=27.6 \pm 6.8 \mathrm{fm}^{-1}$. The results of our calculations, 33.2 and $29.4 \mathrm{fm}^{-1}$, are close to this reconsidered value.

The $\mathcal{R}_{M C M}$ values for $3 / 2_{1}^{+}$, calculated with $\mathrm{V} 2$ and $\mathrm{MN}$, differ only by $1 \%$ and this value, $\mathcal{R}_{M C M}=3.8$, is smaller than the analytical estimate $\mathcal{R}_{0}=4.23 \pm 0.15$ from Eq. (8) but larger than the single-particle value $\mathcal{R}_{\text {s.p. }}=3.62 \pm 0.03$. This difference must originate purely to the charge symmetry breaking due to the Coulomb interaction since the parameters $m$ and $u$ of nuclear NN potentials differ less than by half of a per cent in the mirror $3 / 2^{+}$states. The Coulomb effects should be also responsible for $3 \%$ difference in mirror spectroscopic factors and for deviation of $\left(b_{p} / b_{n}\right)^{2}$ from the single-particle value $\mathcal{R}_{\text {s.p. }}=3.62 \pm 0.03$.

$$
\text { 5. }{ }^{17} \mathrm{~F}-{ }^{17} \mathrm{O}
$$

To describe ${ }^{17} \mathrm{~F}$ and ${ }^{17} \mathrm{O}$, we use single-channel twocluster models ${ }^{16} \mathrm{O}+\mathrm{n}$ and ${ }^{16} \mathrm{O}+\mathrm{p}$ from Refs. [9, 13]. To reproduce simultaneously the proton and neutron separation energies in ${ }^{17} \mathrm{~F}$ and ${ }^{17} \mathrm{O}$, less than $1 \%$ difference in the NN potential parameters in mirror states is required.

The ANCs calculated with V2 are on average 13-14\% larger than those obtained with the MN potential (see Table I). However, the ratio $\mathcal{R}_{M C M}$ of mirror ANCs does not change with NN potential choice in the ground states and differs only by $1 \%$ in the first excited states. The spectroscopic factors are practically insensitive to the NN potential and differ in mirror states by approximately $1 \%$.

In the ${ }^{17} \mathrm{~F}$ and ${ }^{17} \mathrm{O}$ ground states, the $\mathcal{R}_{M C M}=1.19$ value agrees with the single-particle estimate $\mathcal{R}_{\text {s.p. }}=$ $1.21 \pm 0.03$ based on charge symmetry of mirror potential wells and is slightly smaller than prediction $\mathcal{R}_{0}$ $=1.21$ from the analytical formula (8). However, for the first excited state $1 / 2^{+}, \mathcal{R}_{M C M} \approx 730$ is noticably larger than the single-particle value of $702 \pm 4$ and significantly smaller than the analytical value $\mathcal{R}_{0}=837 \pm$ 42. In Sec.IV.A we have shown that, in the presence of charge symmetry of the NN interactions, the $\mathcal{R}_{M C M}$ value, calculated for very small proton separation energies, is approximately the average between $\mathcal{R}_{0}$ and $\mathcal{R}_{\text {s.p. }}$. The $\mathcal{R}_{M C M}$ value of the present section is about $6 \%$ smaller than $\left(\mathcal{R}_{0}+\mathcal{R}_{\text {s.p. }}\right) / 2$ which should be due to the charge symmetry breaking required to reproduce mirror separation energies $\epsilon_{p}$ and $\epsilon_{n}$ in the $1 / 2^{+}$state.

The ratio $b_{p}^{2} / b_{n}^{2}$ of mirror single-particle ANCs squared (733 for V2 and 742 for MN) for the first excited state $1 / 2^{+}$is larger than $\mathcal{R}_{\text {s.p. }}$. This means that in the effective local two-body potential model, the nuclear potential fields for $1 s_{\frac{1}{2}}$ protons and neutrons are slightly different. This contrasts with the situation for $0 d_{\frac{5}{2}}$ proton and neutron in ground states of ${ }^{17} \mathrm{~F}$ and ${ }^{17} \mathrm{O}$, where they can be considered as being placed in the same nuclear potential well.

The results of the calculations described above have been obtained with an oscillator radius of $1.76 \mathrm{fm}$ which reproduces the r.m.s. radius of ${ }^{16} \mathrm{O}$. We have repeated the same calculations with much smaller value of the oscillator radius, $r_{0}=1.5 \mathrm{fm}$, in order to check how $\mathcal{R}_{M C M}$ depends on the wave function of the core ${ }^{16} \mathrm{O}$. With smaller $r_{0},{ }^{16} \mathrm{O}$ has a $38 \%$ smaller r.m.s. radius, the expectation energy of the ${ }^{16} \mathrm{O}$ core is lowered by 20 $\mathrm{MeV}$ and $C_{l}^{2}$ drops by about $40 \%$. However, the $\mathcal{R}_{M C M}$ changes only by $2 \%$ and $5 \%$ for the $5 / 2^{+}$and $1 / 2^{+}$states respectively. This is consistent with the idea behind the formula (8) that the ratio of mirror ANCs depends only 


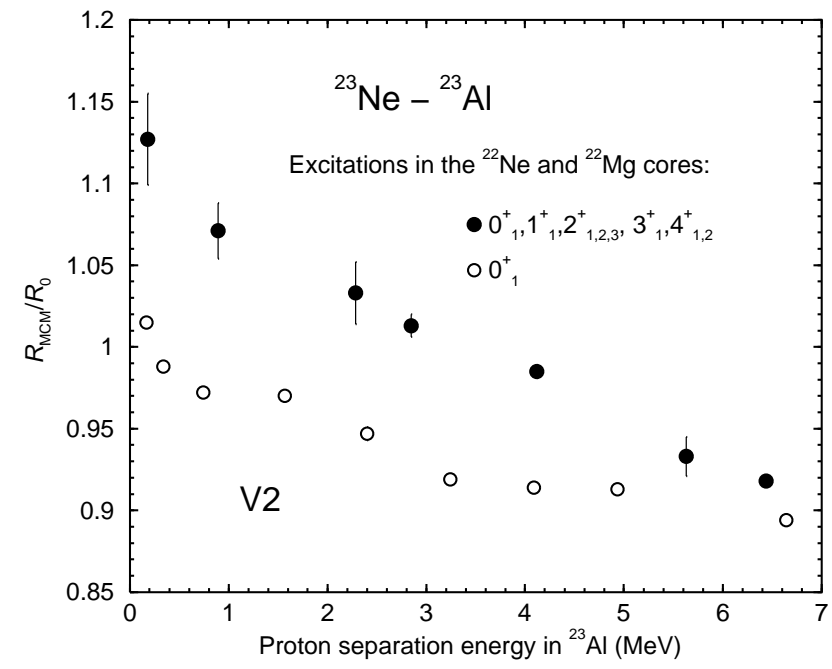

FIG. 2: Ratio $\mathcal{R}_{M C M} / \mathcal{R}_{0}$ for the ${ }^{23} \mathrm{Al}-{ }^{23} \mathrm{Ne}$ mirror pair as a function of the proton separation energy in ${ }^{23} \mathrm{Al}$ calculated without (filled circles) and with (open circles) excitations in the ${ }^{22} \mathrm{Mg}$ and ${ }^{22} \mathrm{Ne}$ cores. The error bars are due to uncertainties in calculating $\mathcal{R}_{0}$, as explained in Sect. IV A. The calculations have been performed with different Majorana parameters $m$ of the Volkov potential V2. Experimental proton separation energy is $0.123 \mathrm{MeV}$.

on the core charge and on the separation energies of mirror proton and neutron.

The experimental value $C_{l}^{2}=0.667 \pm 0.042 \mathrm{fm}^{-1}$ for ${ }^{17} \mathrm{O}_{\text {g.s. }}$. has been determined in Ref. [31]. As already reported in Ref. [9], the MCM calculations with V2 and MN overestimate this value. For the mirror nucleus ${ }^{17} \mathrm{~F}$, the proton ANC has been experimentally determined in Refs. 32, 33, 34, 35, 36] (the ANCs from the data measured in [32, 33] are given in [35]). The $C_{l}^{2}$ values from the first four works, $0.772 \pm 0.19,0.911 \pm 0.082,0.811$ \pm 0.082 and $0.838 \pm 0.05 \mathrm{fm}^{-1}$, agree with each other within the error bars giving the average value of 0.836 $\pm 0.050 \mathrm{fm}^{-1}$. However, the $C_{l}^{2}=1.08 \pm 0.10 \mathrm{fm}^{-1}$ from Ref. [36] is about $30 \%$ larger. The theoretical value $\mathcal{R}_{M C M}=1.19$ agrees well with the averaged experimental value $\mathcal{R}^{\exp }=\left(C_{p}^{\exp } / C_{n}^{e x p}\right)^{2}=1.25 \pm 0.15$ if the $\mathrm{ANC}$ from Ref. [36] is disregarded.

$$
\text { 6. }{ }^{23} \mathrm{Al}-{ }^{23} \mathrm{Ne}
$$

To check if the relation between mirror ANCs is still valid with increasing mass and charge of a mirror pair, we have calculated the overlap integrals $\left\langle\left.{ }^{23} \mathrm{Ne}\left(\frac{5}{2}^{+}\right)\right|^{22} \mathrm{Ne}\left(0^{+}\right)\right\rangle$and $\left\langle\left.{ }^{23} \mathrm{Al}\left(\frac{5}{2}^{+}\right)\right|^{22} \mathrm{Mg}\left(0^{+}\right)\right\rangle$. The latter is relevant to the proton capture reaction ${ }^{22} \mathrm{Mg}(\mathrm{p}, \gamma){ }^{23} \mathrm{Al}$ in novae 37.

We describe ${ }^{23} \mathrm{Al}$ and ${ }^{23} \mathrm{Ne}$ in the multichannel twocluster models ${ }^{22} \mathrm{Mg}+\mathrm{p}$ and ${ }^{22} \mathrm{Ne}+\mathrm{n}$ respectively where the cores ${ }^{22} \mathrm{Mg}$ and ${ }^{22} \mathrm{Ne}$ are in the ground state $0^{+}$and in the excited $1_{1}^{+}, 2_{1,2,3}^{+}, 3_{1}^{+}$and $4_{1,2}^{+}$states. The internal structure of these states is represented by closed $0 s$ and $0 p$ shells and linear combinations of all possible Slater determinants of the $0 d_{\frac{5}{2}}$ shell with the oscillator radius chosen to be $1.7 \mathrm{fm}$. The results of these calculations are presented in Table I.

The calculated ratio $\mathcal{R}_{M C M} \approx 2.95 \times 10^{4}$ is about $12 \%$ higher than both the analytical value $\mathcal{R}_{0}=(2.64 \pm$ $0.03) \times 10^{4}$ and the single-particle value $\mathcal{R}_{\text {s.p. }}=(2.67 \pm$ $0.03) \times 10^{4}$. It is unlikely that such a deviation could come from the $1.5 \%$ difference in the $\mathrm{NN}$ potential parameters needed to reproduce both proton and neutron separation energies in ${ }^{23} \mathrm{Al}$ and ${ }^{23} \mathrm{Ne}$. To exclude this reason, we have computed $\mathcal{R}_{M C M}$ using exactly the same NN interactions in these mirror nuclei. As the result, the divergence between $\mathcal{R}_{M C M}$ and $\mathcal{R}_{0}$ has increased and reached $15 \%$. The agreement between $\mathcal{R}_{M C M}$ and $\mathcal{R}_{0}$ has been restored after we have dropped all channels but one, namely, ${ }^{22} \mathrm{Mg}\left(0_{1}^{+}\right)+\mathrm{p}$ and ${ }^{22} \mathrm{Ne}\left(0_{1}^{+}\right)+\mathrm{n}$, in the wave functions of ${ }^{23} \mathrm{Al}$ and ${ }^{23} \mathrm{Ne}$. By adding and eliminating different configurations (see Table II), we have found out that the main reason for the difference between $\mathcal{R}_{M C M}$ and $\mathcal{R}_{0}$ is the coupling to the $2_{1}^{+}$and $4_{1}^{+}$members of the $0^{+}$ground state rotational band and to the second excited state $4_{2}^{+}$in the ${ }^{22} \mathrm{Ne}$ and ${ }^{22} \mathrm{Mg}$ cores. The spectroscopic factors calculated in the MCM for these core excitations, $0.62,0.75$ and 0.95 respectively, are significantly larger than the spectroscopic factors of $\sim 0.29$ for the cores ${ }^{22} \mathrm{Mg}\left(0_{1}^{+}\right)$and ${ }^{22} \mathrm{Ne}\left(0_{1}^{+}\right)$in their ground states. These spectroscopic factors for mirror overlaps $\left\langle\left.{ }^{23} \mathrm{Ne}\left(\frac{5}{2}^{+}\right)\right|^{22} \mathrm{Ne}\left(0^{+}\right)\right\rangle$and $\left\langle\left.{ }^{23} \mathrm{Al}\left(\frac{5}{2}^{+}\right)\right|^{22} \mathrm{Mg}\left(0^{+}\right)\right\rangle$differ by about $4.5 \%$ and they are reasonably close to the value of 0.34 predicted by the shell model calculations in Ref. 37.

Growing disagreement between $\mathcal{R}_{M C M}$ and $\mathcal{R}_{0}$ with including more core excitations can be explained by increasing role of quadrupole term of $\Delta V_{\text {Coul }}$ in deformed nuclei. This term decreases slowly at large $r$ as $r^{-3}$, giving rise to contributions to Eq. (3) from beyond the nuclear range $R_{N}$, which were ignored in deriving formula (8) for $\mathcal{R}_{0}$. For very small proton separation energies the contribution from nuclear interior to the proton ANC may be even more reduced with increasing orbital momentum $l$ because of the $(\kappa r)^{l}$ behaviour at $r \rightarrow 0$. If this is true, then artificial increase of proton separation energy in ${ }^{23} \mathrm{Al}$ should lead to smaller difference between $\mathcal{R}_{M C M}$ and $\mathcal{R}_{0}$. To check this, we have performed the MCM calculations for V2 with smaller values of $m$. Fig.2 shows that $\mathcal{R}_{M C M} / \mathcal{R}_{0}$ indeed decreases with increasing separation energy $\epsilon_{p}$. The decrease with $\epsilon_{p}$, but to a lesser extent, is also present if all the core excitations are removed (open circles at Fig.2).

$$
\text { 7. }{ }^{27} \mathrm{P}-{ }^{27} \mathrm{Mg}
$$

In this section we study another $s d$-shell mirror pair ${ }^{27} \mathrm{P}-{ }^{27} \mathrm{Mg}$ and the overlap integrals $\left\langle{ }^{27} \mathrm{Mg} \mid{ }^{26} \mathrm{Mg}\right\rangle$ and $\left\langle\left.{ }^{27} \mathrm{P}\right|^{26} \mathrm{Si}\right\rangle$. The latter is relevant to the proton capture 
TABLE II: $\mathcal{R}_{M C M}$ and $\mathcal{R}_{0}$ for the ${ }^{23} \mathrm{Ne}-{ }^{23} \mathrm{Al}$ and ${ }^{27} \mathrm{P}-{ }^{27} \mathrm{Mg}$ mirror pairs calculated with different excitations in the ${ }^{22} \mathrm{Ne}-$ ${ }^{22} \mathrm{Mg}$ and ${ }^{26} \mathrm{Si}-{ }^{26} \mathrm{Mg}$ cores. The calculations have been performed with Volkov potential V2 assuming the same interactions in mirror nuclei and with Majorana parameters $m$ chosen to fit the experimental proton separation energies in ${ }^{23} \mathrm{Al}$ or ${ }^{27} \mathrm{P}$.

\begin{tabular}{lccc}
\hline Core excitations & $\mathcal{R}_{M C M}$ & $\mathcal{R}_{0}$ & $\mathcal{R}_{M C M} / \mathcal{R}_{0}$ \\
\hline & & $\left\langle\left.{ }^{23} \mathrm{Al}\left(\frac{5}{2}^{+}\right)\right|^{22} \mathrm{Mg}\left(0^{+}\right)\right\rangle-\left\langle\left.{ }^{23} \mathrm{Ne}\left(\frac{5}{2}^{+}\right)\right|^{22} \mathrm{Ne}\left(0^{+}\right)\right\rangle$ & 1.02 \\
$0_{1}^{+}$ & $1.86 \times 10^{4}$ & $1.82 \times 10^{4}$ & 1.05 \\
$0_{1}^{+}, 2_{1}^{+}$ & $2.24 \times 10^{4}$ & $2.15 \times 10^{4}$ & 1.09 \\
$0_{1}^{+}, 2_{1}^{+}, 4_{1}^{+}$ & $2.90 \times 10^{4}$ & $2.67 \times 10^{4}$ & 1.10 \\
$0_{1}^{+}, 1_{1}^{+}, 2_{1}^{+}, 4_{1}^{+}$ & $2.93 \times 10^{4}$ & $2.67 \times 10^{4}$ & 1.12 \\
$0_{1}^{+}, 1_{1}^{+}, 2_{1}^{+}, 3_{1}^{+}, 4_{1}^{+}$ & $2.96 \times 10^{4}$ & $2.65 \times 10^{4}$ & 1.12 \\
$0_{1}^{+}, 1_{1}^{+}, 2_{1,2}^{+}, 3_{1}^{+}, 4_{1}^{+}$ & $3.17 \times 10^{4}$ & $2.84 \times 10^{4}$ & 1.12 \\
$0_{1}^{+}, 1_{1}^{+}, 2_{1,2,3}^{+}, 3_{1}^{+}, 4_{1}^{+}$ & $3.21 \times 10^{4}$ & $2.87 \times 10^{4}$ & 1.15 \\
$0_{1}^{+}, 1_{1}^{+}, 2_{1,2,3}^{+}, 3_{1}^{+}, 4_{1,2}^{+}$ & $3.86 \times 10^{4}$ & $3.37 \times 10^{4}$ & 0.95 \\
\hline \hline & & $\left\langle\left.{ }^{27} \mathrm{P}\left(\frac{1}{2}{ }^{+}\right)\right|^{26} \mathrm{Si}\left(0^{+}\right)\right\rangle-\left\langle\left.{ }^{27} \mathrm{Mg}\left(\frac{1}{2}{ }^{+}\right)\right|^{26} \mathrm{Mg}\left(0^{+}\right)\right\rangle$ \\
$0_{1}^{+}$ & 44.04 & 46.3 & 1.03 \\
$0_{1}^{+}, 2_{1}^{+}$ & 46.96 & 45.7 & 1.03 \\
$0_{1}^{+}, 2_{1}^{+}, 4_{1}^{+}$ & 47.08 & 45.8 &
\end{tabular}

reaction ${ }^{26} \mathrm{Si}(\mathrm{p}, \gamma){ }^{27} \mathrm{P}$ in the $r p$-process in the hot stellar hydrogen burning [38].

We describe ${ }^{27} \mathrm{P}$ and ${ }^{27} \mathrm{Mg}$ in the two-cluster models ${ }^{26} \mathrm{Si}+\mathrm{p}$ and ${ }^{26} \mathrm{Mg}+\mathrm{n}$ respectively in which the cores ${ }^{26} \mathrm{Si}$ and ${ }^{26} \mathrm{Mg}$ can be in ground state $0_{1}^{+}$and in first $2_{1}^{+}$ and $4_{1}^{+}$excited states. The internal structure of these states is represented by the Slater determinants composed of $0 s, 0 p$ and $0 d_{\frac{5}{2}}$ single-particle oscillator wave functions with the oscillator radius of $1.7 \mathrm{fm}$.

First, we have studied the dependence of the ratio $\mathcal{R}_{M C M} / \mathcal{R}_{0}$ on core excitations using the assumption of charge-symmetry of the NN interaction. The results, presented in Table II, show that coupling to the configuration with the core in the $2_{1}^{+}$state increases this ratio by $8 \%$. This configuration has a spectroscopic factor of 0.25 which is 3.5 times smaller than that for the ground state. These results have been obtained for the V2 potential, in which the parameter $m$ has been fitted to reproduce the experimental proton separation energy in ${ }^{27} \mathrm{P}$.

With NN interaction different in mirror nuclei, the difference between $\mathcal{R}_{M C M}$ and $\mathcal{R}_{0}$ is $2.5 \%$. The average value $\mathcal{R}_{M C M}=45.0 \pm 0.8$ is larger than the singleparticle estimate $\mathcal{R}_{\text {s.p. }}=40.3 \pm 1.1$, but $\mathcal{R}_{b}=b_{p}^{2} / b_{n}^{2}=$ $40.8 \pm 0.3$ agrees with $\mathcal{R}_{\text {s.p. }}$. This means that potential wells for mirror valence neutron and proton can be considered to be the same. Therefore, the deviation of $\mathcal{R}_{0}$ from $\mathcal{R}_{\text {s.p. }}$ is due to the difference in mirror spectroscopic factors. This difference, $9 \%$ for $\mathrm{V} 2$ and $8 \%$ for $\mathrm{MN}$, is unexpectedly large.

The average value of the spectroscopic factor in ${ }^{27} \mathrm{P}$ and ${ }^{27} \mathrm{Mg}$, which is $\sim 0.85$, is about twice the value predicted by the shell model calculations in [38]. Such a disagreement is most likely caused by neglect of $1 s_{\frac{1}{2}}$ and $0 d_{\frac{3}{2}}$ orbitals in the core wave functions.

\section{SUMMARY AND CONCLUSIONS}

According to the simple analytical formula (8) derived in Ref. [5], the ANCs for mirror virtual nucleon decays are related because of charge symmetry of the NN interaction. This relation is determined only by the separation energies of mirror proton and neutron, the charge of the residual nucleus and the range of its strong interaction with the last nucleon. The ratio of mirror ANCs is not sensitive to the NN potential and details of internal nuclear structure. This ratio should be the same in channels with different spin, or for the same transferred angular momentum $j$.

The MCM calculations of the present paper confirm this general trend. For the mirror pairs considered here, the ratio $\mathcal{R}_{M C M}$ changes by four orders of magnitude as predicted by Eq. (8). Moreover, when charge symmetry of NN interactions is assumed in MCM, $\mathcal{R}_{M C M}$ and $\mathcal{R}_{0}$ for nodeless overlaps are in good agreement even for small separation proton energies. This agreement occurs for both the NN interactions used in calculations. For the overlap $\left\langle{ }^{17} \mathrm{~F}\left(\frac{1}{2}^{+}\right) \mid{ }^{16} \mathrm{O}\right\rangle$ with a node, a judgement about the agreement between $\mathcal{R}_{M C M}$ and $\mathcal{R}_{0}$ is more difficult to make due to uncertainties in the choice of $R_{N}$ to calculate $\mathcal{R}_{0}$. Nevertheless, for very small proton separation energies $\mathcal{R}_{M C M}$ are more closer to $\mathcal{R}_{0}$ rather than to $\mathcal{R}_{\text {s.p. }}$.

The most noticeable disagreement between $\mathcal{R}_{M C M}$ and $\mathcal{R}_{0}$ can be seen for small components of overlap integrals, for example, for $j=1 / 2$ component in $\left\langle\left.{ }^{8} \mathrm{~B}\right|^{7} \mathrm{Be}\right\rangle$. Even in this case, the disagreement is on the level of $8 \%$ if charge symmetry of NN interactions is valid. Stronger disagreement can occur for nuclei with deformed cores. For the ${ }^{23} \mathrm{Al}-{ }^{23} \mathrm{Ne}$ mirror pair, strong coupling to the excited states in the ${ }^{22} \mathrm{Mg}$ and ${ }^{22} \mathrm{Ne}$ cores increase this disagreement up to $15 \%$.

The charge symmetry breaking of the NN interactions, 
TABLE III: Number of nodes $n$, orbital momentum $l$, proton $\left(\epsilon_{p}\right)$ and neutron $\left(\epsilon_{n}\right)$ separation energies (in MeV), single-particle estimate $\mathcal{R}_{\text {s.p. }}$, microscopic calculations $\mathcal{R}_{M C M}$, analytical estimate $\mathcal{R}_{0}$ microscopic calculations for $\mathcal{R}_{b}=C_{p}^{2} S_{n} /\left(C_{n}^{2} S_{p}\right)$ for the mirror pairs from the first column. Average value between calculations with V2 and MN potentials is presented for $\mathcal{R}_{M C M}$ and $\mathcal{R}_{b}$. Charge symmetry breaking of NN interactions is assumed in the MCM calculations.

\begin{tabular}{|c|c|c|c|c|c|c|c|c|c|}
\hline \multirow[t]{2}{*}{ Mirror pair } & \multirow[t]{2}{*}{$J^{\pi}$} & \multirow[t]{2}{*}{$n l$} & \multirow[t]{2}{*}{$\epsilon_{p}$} & \multirow[t]{2}{*}{$\epsilon_{n}$} & \multirow[t]{2}{*}{$\mathcal{R}_{\text {s.p. }}$} & \multirow[t]{2}{*}{$\mathcal{R}_{M C M}$} & \multirow[t]{2}{*}{$\mathcal{R}_{0}$} & \multicolumn{2}{|c|}{$\mathcal{R}_{b}$} \\
\hline & & & & & & & & $j=l-1 / 2$ & $j=l+1 / 2$ \\
\hline${ }^{8} \mathrm{~B}-{ }^{8} \mathrm{Li}$ & $2^{+}$ & $0 p$ & 0.137 & 2.03 & $1.01 \pm 0.01$ & $1.075 \pm 0.013$ & $1.13 \pm 0.01$ & $1.046 \pm 0.014$ & $1.099 \pm 0.001$ \\
\hline${ }^{12} \mathrm{~N}-{ }^{12} \mathrm{~B}$ & $1^{+}$ & $0 \mathrm{p}$ & 0.601 & 3.37 & $1.30 \pm 0.02$ & $1.295 \pm 0.05$ & $1.38 \pm 0.02$ & $1.305 \pm 0.015$ & $1.355 \pm 0.015$ \\
\hline${ }^{13} \mathrm{~N}-{ }^{13} \mathrm{C}^{2 c}$ & $\frac{1}{2}^{-}$ & $0 p$ & 1.944 & 4.95 & $1.168 \pm 0.02$ & $1.135 \pm 0.005$ & $1.198 \pm 0.004$ & & 1.13 \\
\hline${ }^{13} \mathrm{~N}-{ }^{13} \mathrm{C}^{4 c}$ & $\frac{1}{2}^{2}$ & $0 \mathrm{p}$ & 1.944 & 4.95 & $1.168 \pm 0.02$ & $1.19 \pm 0.01$ & $1.198 \pm 0.004$ & & $1.17 \pm 0.01$ \\
\hline${ }^{15} \mathrm{O}-{ }^{15} \mathrm{~N}$ & $\frac{1}{2}^{2}$ & $0 \mathrm{p}$ & 7.297 & 10.8 & $1.51 \pm 0.03$ & $1.477 \pm 0.004$ & 1.48 & $1.506 \pm 0.005$ & $1.40 \pm 0.06$ \\
\hline${ }^{15} \mathrm{O}-{ }^{15} \mathrm{~N}$ & $\frac{3}{2}+$ & $1 \mathrm{~s}$ & 0.507 & 3.53 & $3.62 \pm 0.02$ & $3.805 \pm 0.015$ & $4.23 \pm 0.15$ & & $3.705 \pm 0.025$ \\
\hline${ }^{17} \mathrm{~F}-{ }^{17} \mathrm{O}$ & $\frac{5}{2}^{+}$ & $0 \mathrm{~d}$ & 0.601 & 4.14 & $1.21 \pm 0.03$ & 1.19 & 1.21 & & $1.195 \pm 0.005$ \\
\hline${ }^{17} \mathrm{~F}-{ }^{17} \mathrm{O}$ & $\frac{1}{2}^{+}$ & $1 \mathrm{~s}$ & 0.106 & 3.27 & $702 \pm 4$ & $731 \pm 5$ & $837 \pm 42$ & & $738 \pm 5$ \\
\hline${ }^{23} \mathrm{Al}-{ }^{23} \mathrm{Ne}$ & $\frac{2}{\frac{5}{2}}+$ & $0 \mathrm{~d}$ & 0.123 & 4.42 & $(2.67 \pm 0.03) \times 10^{4}$ & $2.95 \times 10^{4}$ & $(2.63 \pm 0.03) \times 10$ & & $(3.10 \pm 0.01) \times 10^{4}$ \\
\hline${ }^{27} \mathrm{P}-{ }^{27} \mathrm{Mg}$ & $\frac{1}{2}^{2}$ & $1 \mathrm{~s}$ & 0.859 & 6.44 & $40.3 \pm 1.1$ & $45.0 \pm 0.8$ & $44.0 \pm 0.7$ & & $40.8 \pm 0.3$ \\
\hline
\end{tabular}

$4 c$ - four-cluster model

required to reproduce simultaneously the experimental proton and neutron separation energies, reduces $\mathcal{R}_{M C M}$ with respect to $\mathcal{R}_{0}$. This is especially noticeable for twocluster calculations of the ${ }^{13} \mathrm{~N}-{ }^{13} \mathrm{C}$ mirror pair where this effect reaches $6 \%$ (see Table III). These two-cluster calculations require too large odd NN interactions with strong breaking of mirror symmetry. Four-cluster calculations, which do not require strong breaking of mirror symmetry, give much better agreement between $\mathcal{R}_{M C M}$ and $\mathcal{R}_{0}$. Good agreement between $\mathcal{R}_{M C M}$ and $\mathcal{R}_{0}$ also occurs for another 0p overlap $\left\langle\left.{ }^{15} \mathrm{O}\left(\frac{1}{2}^{-}\right)\right|^{14} \mathrm{~N}\right\rangle$. However with decreasing proton separation energy, for example for ${ }^{8} \mathrm{~B}-{ }^{8} \mathrm{Li}$ and ${ }^{12} \mathrm{~N}-{ }^{12} \mathrm{~N}$ mirror pairs, this agreement deteriorates and the deviations reach $6 \%$.

For other nodeless overlaps considered here, the agreement between $\mathcal{R}_{M C M}$ and $\mathcal{R}_{0}$ depends on the deformation of the residual nucleus. In the absence of strong core excitations (the ${ }^{17} \mathrm{~F}\left(\frac{5}{2}^{+}\right)-{ }^{17} \mathrm{O}\left(\frac{5}{2}^{+}\right)$case) the agreement between $\mathcal{R}_{M C M}$ and $\mathcal{R}_{0}$ is good, however strong coupling to excited states of the core may noticably increase $\mathcal{R}_{M C M}$, for example in ${ }^{23} \mathrm{Al}\left(\frac{5}{2}^{+}\right)-{ }^{23} \mathrm{Ne}\left(\frac{5}{2}^{+}\right)$. For overlaps with one node and a loosely bound proton the situation is opposite. $\mathcal{R}_{M C M}$ and $\mathcal{R}_{0}$ are in good agreement if core excitations are present $\left({ }^{27} \mathrm{P}\left(\frac{5}{2}^{+}\right)-{ }^{27} \mathrm{Mg}\left(\frac{5}{2}^{+}\right)\right)$, otherwise $\mathcal{R}_{M C M}$ is smaller than $\mathcal{R}_{0}$ (as in ${ }^{15} \mathrm{O}\left(\frac{3}{2}^{+}\right)$and $\left.{ }^{17} \mathrm{~F}\left(\frac{1}{2}^{+}\right)\right)$.

Our investigation of mirror symmetry of spectroscopic factors has shown that the spectroscopic factors for small components of one-nucleon overlaps can differ up to $20 \%$. For large components of overlaps the mirror spectroscopic factors are almost the same: the spectroscopic factors $S_{l}=S_{l, l-\frac{1}{2}}+S_{l, l+\frac{1}{2}}$ for 0p-shell mirror overlaps may differ up to $3 \%$. For single-particle mirror nuclei ${ }^{17} \mathrm{~F}$ and ${ }^{17} \mathrm{O}$, the spectroscopic factors are the same, while for nuclei in the middle of the sd-shell, mirror spectroscopic factors may differ by up to $9 \%$.

The microscopic calculations of single-particle ANCs $b_{l j}=C_{l j} S_{l j}^{-1 / 2}$ and their ratio squared $\mathcal{R}_{b}$ for mirror overlaps are presented in Table III where they are compared to the single-particle estimates based on assumption of charge symmetry of mirror potential wells. This comparison shows that the concept of mirror symmetry of potential wells is valid only for $j=1 / 2$ component in the ${ }^{12} \mathrm{~N}-{ }^{12} \mathrm{~B}$ and ${ }^{15} \mathrm{O}\left(\frac{1}{2}^{-}\right)-{ }^{15} \mathrm{~N}\left(\frac{1}{2}^{-}\right)$mirror pairs, in the Od nuclei ${ }^{17} \mathrm{~F}\left(\frac{5}{2}^{+}\right)$and ${ }^{17} \mathrm{O}\left(\frac{5}{2}^{+}\right)$and for four-cluster calculations of ${ }^{13} \mathrm{~N}-{ }^{13} \mathrm{C}$. For all other overlap integrals this assumption is not valid. It is interesting that for first excited 1 s-states in ${ }^{17} \mathrm{~F}$ and ${ }^{17} \mathrm{O}$, which are supposed to be good single-particle nuclei, $\mathcal{R}_{b}$ significantly differs from $\mathcal{R}_{\text {s.p. }}$. This means that stronger penetration of the valence $1 \mathrm{~s}$ neutron inside the ${ }^{16} \mathrm{O}$ core perturbs the mean field in greater extent than the mirror proton leading to mirror symmetry breaking in single-particle potential wells.

The assumption that in mirror nuclei both mirror potential wells and mirror spectroscopic factors are equal is valid only for four-cluster model calculations of ${ }^{13} \mathrm{~N}$ $-{ }^{13} \mathrm{C}$ and for ground states of ${ }^{17} \mathrm{~F}-{ }^{17} \mathrm{O}$. However, the deviation between $\mathcal{R}_{\text {s.p. }}$, obtained with this assumption, and microscopic calculations in most cases is not strong, being of the the same order as $\mathcal{R}_{0} / \mathcal{R}_{M C M}$.

The predictions from MCM can be used to calculate proton ANCs using experimentally determined neutron ANCs and vice versa. As an example, let us calculate ANCs for ${ }^{8} \mathrm{~B}$ from experimentally determined values $C_{1 \frac{3}{2}}^{2}\left({ }^{8} \mathrm{Li}\right)=0.384 \pm 0.038 \mathrm{fm}^{-1}$ and $C_{1 \frac{1}{2}}^{2}\left({ }^{8} \mathrm{Li}\right)=0.048$ $\pm 0.006 \mathrm{fm}^{-1}$ from Ref. [18]. With $\mathcal{R}_{\frac{3}{2}}$ and $\mathcal{R}_{\frac{1}{2}}$ values from Table I we get that $C_{1}^{2}\left({ }^{8} \mathrm{~B}\right)$ is $0.460 \pm 0.048 \mathrm{fm}^{-1}$ 
for V2 and $0.471 \pm 0.048 \mathrm{fm}^{-1}$ for MN. This values give the astrophysical $S$-factor of the ${ }^{7} \mathrm{Be}(\mathrm{p}, \gamma)^{8} \mathrm{~B}$ reaction at zero energy $S_{17}(0)=17.8 \pm 1.7 \mathrm{eV} \cdot \mathrm{b}$ for $\mathrm{V} 2$ and $18.2 \pm$ $1.8 \mathrm{eV} \cdot \mathrm{b}$ for the MN. The difference between these two calculations is only $2 \%$.

Finally, if theoretical predictions for the ratio between mirror ANCs are not available, simultaneous consideration of analytical formula (8) and of single-particle estimate $\mathcal{R}_{\text {s.p. }}$ can be used. Based on our calculations, the average between these values may be a reasonably good approximation if the core is not strongly deformed. Strong core polarization effects can increase this ratio.
The largest increase, calculated in the present paper, is $12 \%$.

\section{Acknowledgements}

N.K.T. is grateful to Professors R.C. Johnson and I.J. Thompson for fruitful discussions and useful comments concerning this manuscript. Support from the UK EP$\mathrm{SRC}$ via grant GR/T28577 is gratefully acknowledged.
[1] H.M. Xu, C.A. Gagliardi, R.E. Tribble, A.M. Mukhamedzhanov and N.K. Timofeyuk, Phys. Rev. Lett. 73, 2027 (1994).

[2] N.K. Timofeyuk, Nucl. Phys. A632, 38 (1998).

[3] N.K. Timofeyuk and S.B. Igamov, Nucl. Phys. A713, 217 (2003).

[4] L.Trache, A.Azhari, F.Carstoiu, H.L.Clark, C.A.Gagliardi, Y.-W.Lui, A.M.Mukhamedzhanov, X.Tang, N.Timofeyuk, R.E.Tribble, Phys.Rev. C 67, 062801(R) (2003)

[5] N.K. Timofeyuk, R.C. Johnson and A.M. Mukhamedzhanov, Phys. Rev. Lett. 91, 232501 (2003).

[6] L.D. Blokhintsev, I. Borbely and E.I. Dolinskii, Sov. J. Part. Nucl. 8, 485 (1977).

[7] A.M. Mukhamedzhanov and N.K. Timofeyuk, Sov. J. Nucl. Phys. 51, 431 (1990) [Yad. Fiz. 51 (1990) 679].

[8] D. Baye, P.-H. Heenen and M. Libert-Heinemann, Nucl. Phys. A291, 230 (1977).

[9] D. Baye and N.K. Timofeyuk, Phys. Lett. B293, 13 (1992).

[10] A.B. Volkov, Nucl. Phys. 74, 33 (1965).

[11] D.R. Thompson, M. LeMere and Y.C. Tang, Nucl. Phys. A286, 53 (1977).

[12] D. Baye and N. Pecher, Bull Sc. Acad. Roy. Belg. 67 835, (1981).

[13] D. Baye, P. Descouvemont and M. Hesse, Phys. Rev. C 58, 545 (1998)

[14] P. Descouvemont, Phys. Rev. C 70, 065802 (2004)

[15] N.K. Timofeyuk, D. Baye and P. Descouvemont, Nucl. Phys. A620, 29 (1997).

[16] A. Azhari et al, Phys. Rev. C 63, 055803 (2001).

[17] L. Trache, F.Carstoiu, C.A. Gagliardi and R.E. Tribble, Phys. Rev. Lett. C 87, 271102 (2001).

[18] L. Trache et al, Phys. Rev. C 67, 062801(R) (2003).

[19] P. Descouvemont, Nucl. Phys. A646, 261 (1999)
[20] Z.H. Liu, C.J. Lin, H.Q. Zhang, Z.C. Li, J.S. Zhang, Y.W. Wu, F. Yang, M. Ruan, J.C. Liu, S.Y. Li and Z.H. Peng, Phys. Rev. C 64, 034312 (2001).

[21] X. Tang et al, Phys. Rev. C 67, 015804 (2003).

[22] M. Dufour and P. Descouvemont, Phys. Rev. C 56, 1831 (1997)

[23] H.P. Gubler, G.R. Plattner, I. Sick, A. Traber and W. Weiss, Nucl. Phys. A284, 114 (1977)

[24] S.S. Kajumov, A.M. Mukhamedzhanov and R. Yarmukhamedov, Z. Phys. A331, 315 (1988)

[25] G.D. Putt, Nucl. Phys. A161, 547 (1971)

[26] S.A. Goncharov, J. Dobesh, E.I. Dolinskii, A.M. Mukhamedzhanov and J. Cejpek, Sov. J. Nucl. Phys. 35, 383 (1982) [Yad. Fiz. 35, 662 (1982)].

[27] I.R. Gulamov, A.M. Mukhamedzhanov and G.K. Nie, Phys. At. Nucl. 58 (1995) 1689

[28] S. Cohen, D. Kurath, Nucl. Phys. 101, 1 (1967).

[29] A.M. Mukhamedzhanov et al, Phys. Rev. C 67, 065804 (2003).

[30] P.F. Bertone et al, Phys. Rev. C 66, 055804 (2002).

[31] S. Burzynski et al, Nucl. Phys. A399, 230 (1983).

[32] H.T. Fortune et al, Phys. Rev. 12, 1723 (1975).

[33] J. Vernotte et al, Nucl. Phys. A571, 1 (1994).

[34] S.V. Artemov et al, Phys. At. Nucl. 59, 428 (1996)

[35] S.V. Artemov et al, Phys. At. Nucl. 63, 1763 (2000).

[36] C.A. Gagliardi et al, Phys. Rev. C 59, 1149 (1999).

[37] J.A. Caggiano et al, Phys. Rev. C 64, 025802 (2001).

[38] H.Herndl, J.Görres, M.Wiescher, B.A. Brown and L. VanWormer, Phys. Rev. C 52, 1078 (1995).

[39] We have found a mistake in the phase factor of the function $\varphi_{l}(i \kappa r)$ in Refs. [2, 5, 7]. The corrected phase factor is given in Eq. (4) of the present work. This mistake does not influence the published previously ANCs, spectroscopic factors or shapes of overlap integrals. 